\title{
Practical Aspects of the Frequency Domain Approach for Aircraft System Identification
}

\author{
Eugene A. Morelli ${ }^{1}$ and Jared A. Grauer ${ }^{2}$ \\ NASA Langley Research Center, Hampton, Virginia, 23681
}

\begin{abstract}
Practical aspects of the frequency-domain approach for aircraft system identification are explained and demonstrated. Topics related to experiment design, flight data analysis, and dynamic modeling are included. For demonstration purposes, simulated time series data and simulated flight data from an F-16 nonlinear simulation with realistic noise are used. This approach enables detailed evaluations of the techniques and results, because the true characteristics of the data and aircraft dynamics are known for the simulated data. Analytical techniques and practical considerations are examined for the finite Fourier transform, nonparametric frequency response estimation, parametric modeling in the frequency domain, experiment design for frequency-domain modeling, data analysis and modeling in the frequency domain, and real-time calculations. Flight data from a subscale jet transport aircraft are used to demonstrate some of the techniques and technical issues.
\end{abstract}

\begin{tabular}{|c|c|c|}
\hline & & Nomenclature \\
\hline$a_{x}, a_{y}, a_{z}$ & $=$ & body-axis translational accelerometer measurements, $\mathrm{g}$ \\
\hline $\boldsymbol{A}, \boldsymbol{B}, \boldsymbol{C}, \boldsymbol{D}$ & $=$ & linear dynamic system matrices \\
\hline$b$ & $=$ & wing span, $\mathrm{ft}$ \\
\hline $\bar{c}$ & $=$ & wing mean aerodynamic chord, $\mathrm{ft}$ \\
\hline$C_{X}, C_{Y}, C_{Z}$ & $=$ & body-axis nondimensional aerodynamic force coefficients \\
\hline$C_{l}, C_{m}, C_{n}$ & $=$ & body-axis nondimensional aerodynamic moment coefficients \\
\hline $\mathcal{F}[\cdot]$ & $=$ & Fourier transform \\
\hline$I_{x}, I_{y}, I_{z}, I_{x z}$ & $=$ & mass moments of inertia, slug- $\mathrm{ft}^{2}$ \\
\hline$j$ & $=$ & imaginary number $=\sqrt{-1}$ \\
\hline$m$ & $=$ & aircraft mass, slug \\
\hline$p, q, r$ & $=$ & body-axis roll, pitch, and yaw rates, $\mathrm{rad} / \mathrm{s}$ or $\mathrm{deg} / \mathrm{s}$ \\
\hline $\bar{q}$ & $=$ & dynamic pressure, $\mathrm{lbf} / \mathrm{ft}^{2}$ \\
\hline$S$ & $=$ & wing reference area, $\mathrm{ft}^{2}$ \\
\hline$T$ & $=$ & maneuver length, $\mathrm{s}$ \\
\hline$V$ & $=$ & true airspeed, $\mathrm{ft} / \mathrm{s}$ \\
\hline$\alpha$ & $=$ & angle of attack, rad or deg \\
\hline$\beta$ & $=$ & sideslip angle, rad or deg \\
\hline$\delta_{s}, \delta_{e}, \delta_{a}, \delta_{r}$ & $=$ & stabilator, elevator, aileron, and rudder deflections, rad or deg \\
\hline$\phi, \theta, \psi$ & $=$ & Euler roll, pitch, and yaw angles, rad or deg \\
\hline$\Sigma$ & $=$ & covariance matrix \\
\hline subscripts & & \\
\hline$c g$ & $=$ & center of gravity \\
\hline$o$ & $=$ & reference value or base term \\
\hline
\end{tabular}

\footnotetext{
${ }^{1}$ Research Engineer, Dynamic Systems and Control Branch, MS 308, AIAA Associate Fellow

${ }^{2}$ Research Engineer, Dynamic Systems and Control Branch, MS 308, AIAA Senior Member
} 


\section{superscripts}

$\begin{array}{lll}T & =\text { transpose } \\ & =\text { estimate } \\ \sim & =\text { time derivative } \\ * & =\text { Fourier transform } \\ + & =\text { complex conjugate } \\ -1 & =\text { complex conjugate transpose } \\ \underline{\text { acronyms }} & =\text { matrix inverse } \\ \text { DFT } & =\text { Discrete Fourier Transform } \\ \text { FFT } & =\text { Fast Fourier Transform } \\ \text { SIDPAC } & =\text { System IDentification Programs for AirCraft }\end{array}$

\section{Introduction}

$\mathrm{F}$ LIGHT data analysis and dynamic modeling can be carried out in the time domain or in the frequency domain ${ }^{1}$. Frequency-domain analysis has many advantages, such as providing particular types of physical insight, direct applicability to common control system design approaches, robustness to noise, and lower dimensionality for model parameter estimation, among others ${ }^{1-6}$. Frequency-domain techniques based on spectral estimation ${ }^{1,2,6}$, output error $^{1-4}$, and equation error ${ }^{1,2,4,5}$ have been applied very successfully for flight test data analysis and linear dynamic modeling. The basis for all of these frequency domain methods is the finite Fourier transform, which is the analytical tool for transforming time-domain data to the frequency domain. Any errors in the transformation from the time domain to the frequency domain will affect the accuracy of the raw data in the frequency domain, which in turn impacts data analysis and modeling results obtained using frequency-domain methods.

The purpose of this work is to examine practical issues encountered when analyzing data in the frequency domain, from the standpoint of how the data are changed by transformation to the frequency domain, as well as the how data analysis and modeling results should be computed and interpreted in the frequency domain. The intent is to provide a solid foundation of understanding for applying frequency-domain methods to flight data analysis and modeling. In addition, important extensions of conventional frequency-domain methods are described and demonstrated, along with comparisons of various approaches to solving particular problems in the frequency domain.

Following a brief description of the F-16 nonlinear simulation ${ }^{1,7}$ and the T-2 subscale jet transport aircraft ${ }^{8-11}$, the paper is arranged as a series of technical topics related to flight data analysis and modeling in the frequency domain. For demonstration purposes, simulated time series data and simulated flight data from an F-16 nonlinear simulation with realistic noise were used. This approach enabled detailed evaluations of the techniques and results, because the true characteristics of the data and aircraft dynamics were known for the simulated data. Measured flight data from the T-2 subscale jet transport aircraft were also used to illustrate and explain some of the topics.

All of the tools used to generate the results shown in this paper are available in a MATLAB ${ }^{\circledR}$ software package called SIDPAC (ㅁystem IDentification Programs for AirCraft). SIDPAC is associated with Ref. [1], and is available in the US at no $\operatorname{cost}^{12}$. The SIDPAC software toolbox was developed at NASA Langley, and is continually expanded and improved. SIDPAC has been applied successfully to a wide variety of flight and wind tunnel experiments at NASA Langley and elsewhere, and is used at more than 100 organizations in government, industry, and academia ${ }^{13}$.

\section{Aircraft}

\section{A. F-16 Nonlinear Aircraft Simulation}

The F-16 is a single-seat, multi-role fighter with a blended wing / body and a cropped delta wing planform with leading edge sweep of $40 \mathrm{deg}$. Thrust is provided by one General Electric F110-GE-100 or Pratt \& Whitney F100PW-220 afterburning turbofan engine mounted in the rear fuselage. Figure 1 is a photograph of the F-16 in flight. Aircraft geometry and nominal mass properties are given in Table 1.

The F-16 was modeled with controls for throttle $\delta_{t h}$, stabilator $\delta_{s}$, aileron $\delta_{a}$, and rudder $\delta_{r}$. Speed brake and flaps were assumed fixed at zero deflection. Throttle deflection was limited to the range $0 \leq \delta_{t h} \leq 1$, stabilator 
deflection was limited to $-25^{\circ} \leq \delta_{s} \leq 25^{\circ}$, aileron deflection was limited to $-21.5^{\circ} \leq \delta_{a} \leq 21.5^{\circ}$, and rudder deflection was limited to $-30^{\circ} \leq \delta_{r} \leq 30^{\circ}$. These limits represent the physicals stops.

Nondimensional aerodynamic force and moment coefficient data were derived from a low-speed static wind tunnel test and a dynamic forced oscillation wind tunnel test ${ }^{14}$, both conducted with a $16 \%$ scale model of the F-16. The aerodynamic database applies to the F-16 flown out of ground effect, with landing gear retracted, and no external stores. Static aerodynamic data were in tabular form as a function of angle of attack and sideslip angle over the ranges $-10^{\circ} \leq \alpha \leq 45^{\circ}$ and $-30^{\circ} \leq \beta \leq 30^{\circ}$, respectively. Dynamic data were provided in tabular form at zero sideslip angle over the angle of attack range $-10^{\circ} \leq \alpha \leq 45^{\circ}$. Dependence of the nondimensional coefficients on $\dot{\alpha}$ was included with the $q$ dependencies, because of the manner in which the data were collected in the wind tunnel.

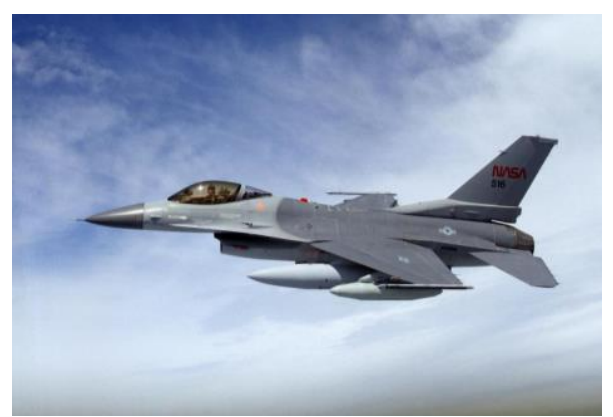

Figure 1. F-16 Aircraft

Credit: NASA Langley Research Center

The engine model was based on ground test data in tabular form, with thrust given as a function of altitude, Mach number, and engine power level. Engine power level was computed from throttle position and throttle gearing, including first-order lag dynamics for the thrust response.

The F-16 nonlinear simulation was programmed completely in MATLAB ${ }^{\circledR}$. Full nonlinear equations of motion, including turbine engine gyroscopic effects, were used. Complete details on the F-16 nonlinear simulation can be found in Ref. [7] or in Appendix D of Ref. [1].

\section{B. T-2 Subscale Jet Transport Aircraft}

The T-2 aircraft is a 5.5-percent dynamically-scaled model of a generic commercial twin-engine jet transport aircraft. A photograph of the aircraft in flight is shown in Figure 2. The aircraft has twin jet engines mounted under the wings and retractable tricycle landing gear. Aircraft geometry and nominal mass properties are given in Table 1. Further information on the T-2 subscale jet transport aircraft and associated flight test operations can be found in Refs. [8]-[11].

Control surfaces on the T-2 aircraft are left and right ailerons, left and right inboard and outboard elevators, upper and lower rudders, left and right inboard and outboard trailingedge flaps, and left and right inboard and outboard spoilers, for a total of 16 independent control surfaces. For the flight data

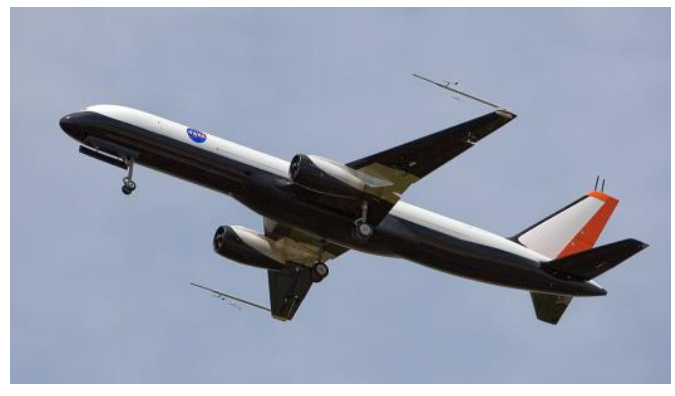

Figure 2. T-2 subscale jet transport aircraft Credit: NASA Langley Research Center used in this work, only the elevators, ailerons, and rudders were deflected. The individual elevator surfaces were moved together as a single elevator surface, and similarly for the rudders. Left and right ailerons were deflected asymmetrically, in the conventional way. Definitions of control surface deflections are given below. Trailing edge down is positive deflection for wing and elevator surfaces, and trailing edge left is positive for rudder surfaces.

$$
\delta_{e}=\frac{1}{4}\left(\delta_{e_{l o}}+\delta_{e_{l i}}+\delta_{e_{r i}}+\delta_{e_{r o}}\right) \quad \delta_{a}=\frac{1}{2}\left(\delta_{a_{r}}-\delta_{a_{l}}\right) \quad \delta_{r}=\frac{1}{2}\left(\delta_{r_{u}}+\delta_{r_{l}}\right)
$$

The aircraft can be flown by a safety pilot using direct visual contact and conventional radio control. A research pilot executed the flight test maneuvers from inside a mobile control room, using a synthetic vision display generated from telemetry data and a local terrain database, along with video from a camera in the nose of the aircraft. Inputs from the research pilot and a ground-based flight control system produced control surface commands which were transmitted by telemetry to the aircraft.

The flight control system has the capability to inject automated control surface perturbations to excite the aircraft dynamic response for modeling purposes. These control surface perturbations can have arbitrary waveforms, and can be applied to multiple control surfaces individually or simultaneously. The perturbations are summed with pilot and feedback control commands in the flight control system, just before the limiting on control surface actuator 
command rates and positions. Typically, the research pilot flies the aircraft to the desired flight condition(s), then initiates the automated control surface perturbations with a trigger switch on the throttle control.

The T-2 aircraft was equipped with a micro-INS, which provided 3-axis translational accelerometer measurements, angular rate measurements, estimated attitude angles, and GPS velocity and position. Air data probes attached to booms mounted on each wingtip (visible in Fig. 2) measured angle of attack, sideslip angle, static pressure, and dynamic pressure. Measurements from static pressure sensors and ambient temperature sensors were used to compute air density and altitude. Engine rotational speeds were measured and used as inputs to an engine model to compute thrust. The engine model was identified from ground test data, with ram drag adjustments determined from flight data. Potentiometers mounted on the rotation axes of the control surfaces measured control surface deflections. Mass properties were computed based on measured fuel flow, pre-flight weight and balance, and inertia measurements done on the ground for the aircraft without fuel. Pilot stick and rudder pedal positions and throttle position were also measured and recorded. Data from onboard sensors were telemetered to the ground in real time. Sampling rate for the flight data was $200 \mathrm{~Hz}$, downsampled to $50 \mathrm{~Hz}$ for data analysis and modeling.

\section{Transforming Time-Domain Data to the Frequency Domain}

The analytical tool for transforming data from the time domain to the frequency domain is the finite Fourier transform, defined by

$$
\tilde{x}(\omega) \equiv \int_{0}^{T} x(t) e^{-j \omega t} d t
$$

where

$$
\omega=2 \pi f
$$

These relationships show that the finite Fourier transform $\tilde{x}(\omega)$ can be interpreted as a coefficient in an expansion of $x(t)$ in terms of basis functions $e^{-j \omega t}=\cos \omega t-j \sin \omega t$ for each frequency $\omega$, or as a measure of the similarity of $x(t)$ to the functions $e^{-j \omega t}$ for each frequency $\omega$.

When the time function $x(t)$ is sampled at constant discrete time intervals $\Delta t$, the finite Fourier transform can be approximated by

$$
\tilde{x}(\omega) \approx \Delta t \sum_{i=0}^{N-1} x(i) e^{-j \omega i \Delta t}
$$

or

$$
\tilde{x}(f) \approx \Delta t \sum_{i=0}^{N-1} x(i) e^{-j 2 \pi f i \Delta t}
$$

where the time length of the measured data is $T=(N-1) \Delta t$ and

$$
t_{i}=i \Delta t \quad x(i) \equiv x\left(t_{i}\right)=x(i \Delta t) \quad i=0,1,2, \ldots, N-1
$$

Note that the total number of data points in the time domain is $N$, and the time index $i$ starts at 0 . Equation (4a) is a simple Euler approximation for the finite Fourier transform of Eq. (2), using $N$ discrete samples of the continuous time function $x(t)$. For a conventional finite Fourier transform, the frequencies are chosen as

$$
f_{k}=\frac{k}{N \Delta t} \quad k=0,1,2, \ldots, N-1
$$

or

$$
\omega_{k}=2 \pi f_{k}=2 \pi \frac{k}{N \Delta t} \quad k=0,1,2, \ldots, N-1
$$


Using these discrete frequencies, the approximation to the finite Fourier transform in Eq. (4b) becomes

$$
\tilde{x}(k) \approx \Delta t \sum_{i=0}^{N-1} x(i) e^{-j(2 \pi k / N) i} \quad k=0,1,2, \ldots, N-1
$$

and the inverse is

$$
x(i) \approx \frac{1}{N \Delta t} \sum_{k=0}^{N-1} \tilde{x}(k) e^{j(2 \pi k / N) i} \quad i=0,1,2, \ldots, N-1
$$

Often Eqs. (7) are written for the normalized sampling interval $\Delta t=1$. The result is called the discrete Fourier transform, defined by

$$
X(k) \equiv \sum_{i=0}^{N-1} x(i) e^{-j(2 \pi k / N) i} \quad k=0,1,2, \ldots, N-1
$$

which has the inverse

$$
x(i)=\frac{1}{N} \sum_{k=0}^{N-1} X(k) e^{j(2 \pi k / N) i} \quad i=0,1,2, \ldots, N-1
$$

Equations (8) are called the discrete Fourier transform (DFT) pair.

In general, the DFT coefficients are complex numbers, with real and imaginary parts. If $x(i), i=0,1,2, \ldots, N-1$, is a sequence of real numbers, as is the case for flight test data, it can be seen from Eq. (8a) that

$$
X(N-k)=X^{*}(k)
$$

It follows that the transformed data has conjugate symmetry about the value of $k$ corresponding to the Nyquist frequency $1 /(2 \Delta t)$. Because of this, it suffices to compute the finite Fourier transform for only the first $M$ frequencies defined in Eqs. (6), where

$$
M=\left\{\begin{array}{cc}
N / 2+1 & \text { for } N \text { even } \\
(N+1) / 2 & \text { for } N \text { odd }
\end{array}\right.
$$

These values for $M$ represent the fundamental limitation that frequencies contained in a sampled time history must fall within the frequency band $\left[0, f_{N}\right]$, where $f_{N}=1 /(2 \Delta t)$ is the Nyquist frequency, defined as half the sampling frequency.

Direct computation of the DFT using Eq. (8a) is not efficient computationally. A numerically efficient method for computing the DFT is the Fast Fourier Transform (FFT) algorithm ${ }^{15}$, which is used as the basis for many Fourier transform computations.

\section{A. Frequency Resolution}

For the conventional discrete Fourier transform in Eq. (8a), the quantity involving the analysis frequencies is

$$
e^{-j(2 \pi k / N)} \equiv e^{-j \theta_{k}} \equiv z_{k} \quad k=0,1,2, \ldots, N-1
$$

which can be represented by evenly-spaced points around the unit circle in the complex plane, see Fig. 3. This frequency selection was developed so that the entire available frequency band up to the Nyquist frequency would be covered, and the discrete Fourier transform calculations could be done with computational efficiency using the FFT.

For a uniform sampling interval $\Delta t$, Eq. (6a) shows that the frequency resolution depends on the time length of the measured data $T$ and is approximately equal to $1 / T \mathrm{~Hz}$. Efficient FFT algorithms for computing the DFT use this particular selection of frequencies, which means that the analysis frequencies cannot be selected arbitrarily for a given data record length. If the time length $T$ is short, this frequency resolution can be unacceptably coarse, particularly for rigid-body aircraft dynamic modeling, where the frequency band containing the dynamic response 
might be $[0,1] \mathrm{Hz}$ for a full-scale aircraft. Coarse frequency resolution can omit important data features in the frequency domain. For typical flight data with $50 \mathrm{~Hz}$ sampling rate, most of the processing involved in the FFT calculation of the discrete Fourier transform is done for frequencies outside the range of interest.

Adding zeros to the measured time-domain data to artificially increase the data record length, known as zero padding, is sometimes done in an attempt to increase resolution in the frequency domain using the FFT, because the analysis frequencies are tied to the data record length. However, this approach results in interpolation of the frequency-domain data obtained from the original time series, rather than increased resolution in the frequency domain. Zero padding is also sometimes done to make the number of data points in the time domain equal to an integer power of 2 , which is a requirement for maximum speed of FFT algorithms. However, typical post-flight data analysis does not involve tight limitations on computation time, so the speed of FFT algorithms is not needed with modern computers. Furthermore, the simple Euler approximation used in the discrete Fourier transform and the FFT algorithm become increasingly inaccurate as the analysis frequencies and sampling interval increase.

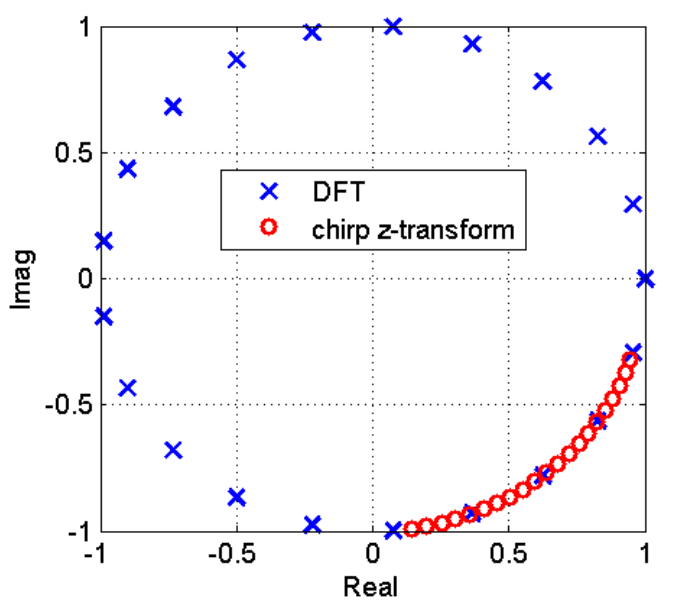

Figure 3. Values of the transform variable $z_{k}=e^{-j \theta_{k}}$

\section{High-Accuracy Finite Fourier Transform with Arbitrary Frequency Resolution}

The finite Fourier transform in Eq. (2) can be computed very accurately with arbitrarily fine frequency resolution using the finite Fourier transform method described in Refs. [1] and [16], and implemented in SIDPAC ${ }^{1,12}$. This approach allows arbitrary choice of both the limits of the frequency band and the frequency resolution, corresponding to the start, end, and spacing of the analysis frequencies, as demonstrated by the circles shown in Fig. 3. The chirp $z$-transform ${ }^{17}$ can be applied to compute the discrete Fourier transform with frequencies finer than $1 / T \mathrm{~Hz}$, but it is more accurate and general to compute the finite Fourier transform in Eq. (2) using the numerical method described in Refs. [1] and [16], and implemented in the SIDPAC code fint.m. This method applies the chirp $z$-transform for improved frequency resolution, but improves the accuracy of the finite Fourier transform using local cubic interpolation of the sampled time series data and special calculations near the endpoints. Using this approach, the value of the finite Fourier transform can be computed at arbitrarily selected frequencies anywhere within the frequency band $\left[0, f_{N}\right]$ with errors on the order of the numerical precision of the computer. This capability provides a solid and versatile underpinning to many frequency domain techniques and renders those methods more accurate and flexible. All finite Fourier transformations in this work were done using this method, unless otherwise specified.

\section{Frequency-Domain Sampling}

To examine frequency-domain sampling, first note that for a simple sinusoid $x(t)=A \sin \left(\omega_{o} t\right)$, the finite Fourier transform can be computed analytically,

$$
\tilde{x}(\omega)=\int_{0}^{t} A \sin \left(\omega_{o} \tau\right) e^{-j \omega \tau} d \tau
$$




$$
\begin{gathered}
=\int_{0}^{t} A \frac{\left(e^{j \omega_{o} \tau}-e^{-j \omega_{o} \tau}\right)}{2 j} e^{-j \omega \tau} d \tau \\
=\frac{A}{2 j} \int_{0}^{t}\left[e^{-j\left(\omega-\omega_{o}\right) \tau}-e^{-j\left(\omega+\omega_{o}\right) \tau}\right] d \tau \\
=\frac{A}{2}\left[\frac{e^{-j\left(\omega-\omega_{o}\right) \tau}}{\left(\omega-\omega_{o}\right)}-\frac{e^{-j\left(\omega+\omega_{o}\right) \tau}}{\left(\omega+\omega_{o}\right)}\right]_{0}^{t} \\
=\frac{A}{2}\left\{\frac{\left[e^{-j\left(\omega-\omega_{o}\right) t}-1\right]}{\left(\omega-\omega_{o}\right)}-\frac{\left.e^{-j\left(\omega+\omega_{o}\right) t}-1\right]}{\left(\omega+\omega_{o}\right)}\right\} \\
\tilde{x}(\omega)=-j \frac{A}{2} t\left\{\frac{e^{-j\left(\omega-\omega_{o}\right) t / 2}\left[e^{-j\left(\omega-\omega_{o}\right) t / 2}-e^{j\left(\omega-\omega_{o}\right) t / 2}\right]}{-2 j\left(\omega-\omega_{o}\right) t / 2}-\frac{e^{-j\left(\omega+\omega_{o}\right) t / 2}\left[e^{-j\left(\omega+\omega_{o}\right) t / 2}-e^{j\left(\omega+\omega_{o}\right) t / 2}\right]}{-2 j\left(\omega+\omega_{o}\right) t / 2}\right. \\
\tilde{x}(\omega)=-j \frac{A}{2} t\left\{\frac{e^{-j\left(\omega-\omega_{o}\right) t / 2} \sin \left[\left(\omega-\omega_{o}\right) t / 2\right]}{\left(\omega-\omega_{o}\right) t / 2}-\frac{e^{-j\left(\omega+\omega_{o}\right) t / 2} \sin \left[\left(\omega+\omega_{o}\right) t / 2\right]}{\left(\omega+\omega_{o}\right) t / 2}\right\} \\
\tilde{x}(\omega)=j \frac{A}{2} t\left\{e^{-j\left(\omega+\omega_{o}\right) t / 2} \operatorname{sinc}\left[\left(\omega+\omega_{o}\right) t / 2\right]-e^{-j\left(\omega-\omega_{o}\right) t / 2} \operatorname{sinc}\left[\left(\omega-\omega_{o}\right) t / 2\right]\right\}
\end{gathered}
$$

Similarly, for $x(t)=A \cos \left(\omega_{o} t\right)$,

$$
\tilde{x}(\omega)=\frac{A}{2} t\left\{e^{-j\left(\omega+\omega_{o}\right) t / 2} \operatorname{sinc}\left[\left(\omega+\omega_{o}\right) t / 2\right]+e^{-j\left(\omega-\omega_{o}\right) t / 2} \operatorname{sinc}\left[\left(\omega-\omega_{o}\right) t / 2\right]\right\}
$$

Because the time length $t$ appears on the right sides of Eqs. (13) and (14), some definitions of the finite Fourier transform include division by the time length to normalize the increasing values of the finite Fourier transform with increasing time, or to examine or compare frequency content for different data record lengths.

Figure 4 shows the analytic finite Fourier transform computed from Eq. (13) for the sinusoid $x(t)=A \sin \left(\omega_{o} t\right)$ with $A=1, \omega_{o}=2 \pi f_{o} / T, f_{o}=10 \mathrm{~Hz}, T=20 \mathrm{~s}$, or $x(t)=\sin (\pi t)$. The scalloping in the analytic finite Fourier transform data, called leakage, is a smearing of frequency components to adjacent frequencies, and is the result of a finite (as opposed to infinite) data record ${ }^{1,15,18,19}$. If the analysis frequencies used in the finite Fourier transform are selected as the harmonic frequencies $2 \pi k / T, k=1,2, \ldots, N-1$, then the frequency sampling occurs at the peak of the major lobe and at the zeros of the true frequency-domain data, as shown by the x markers. Any deviation from these frequencies will sample the true frequency-domain data at different frequencies, as demonstrated by the $o$ markers.

Using harmonic frequencies $2 \pi k / T, k=1,2, \ldots, N-1$, based on the data record length $T$, but evaluating the finite Fourier transform at a time different than $T$ is demonstrated by the x markers in Fig. 5 for $13 \mathrm{~s}$. This is similar in effect to using frequencies different from the harmonic frequencies based on the data record length $T$, and evaluating the finite Fourier transform at time $T$, which was demonstrated by the o markers in Fig. 4.

It follows that when the analysis frequencies are mismatched with the record length $T$ and/or the fundamental period of the time-domain data (the usual practical case), then the frequency sampling will not be the ideal shown by the $\mathrm{x}$ markers in Fig. 4, but rather some other sampling of the true frequency-domain data. The important point is that the underlying frequency-domain characteristic is the same - only the frequency sampling is changed. Equivalently, the view of the underlying frequency-domain characteristic changes according to the frequency sampling. These same statements apply in the practical case where the time series is a sum of various sinusoids with arbitrary amplitudes and phase shifts, rather than the single pure sinusoid with unit amplitude and zero phase shift 
used here for demonstration purposes. In practice, the underlying frequency-domain characteristic will be more complex, but the same analysis applies.

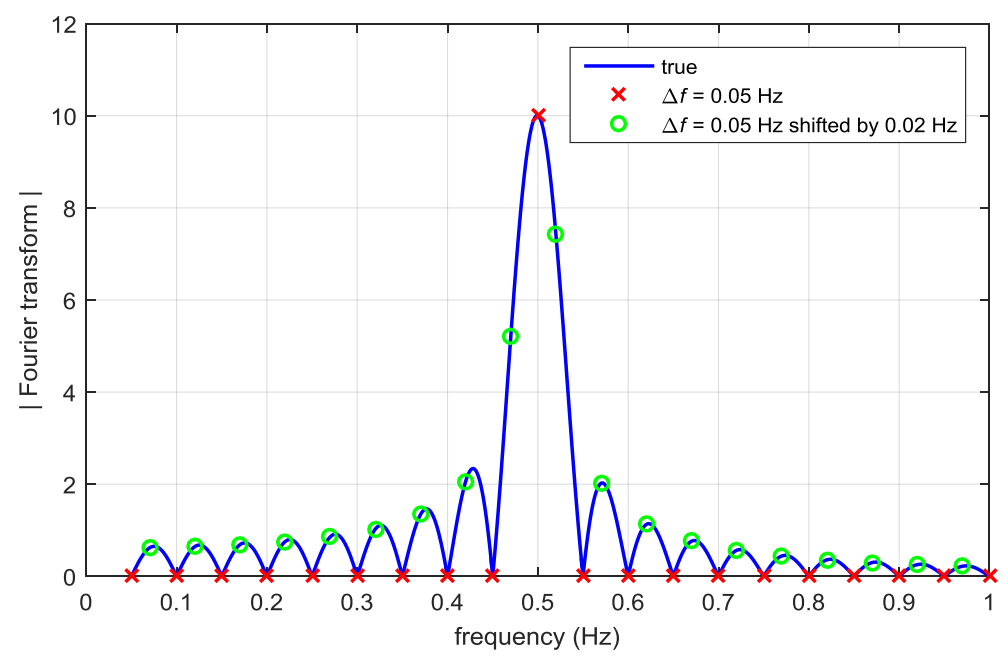

Figure 4. Frequency sampling $\Delta f=1 /(20 \mathrm{~s})=0.05 \mathrm{~Hz}$ for $x(t)=\sin (\pi t)$ at $20 \mathrm{~s}$

Figure 5 demonstrates the case where the data record length is less than the value $T$ used for the resolution of the analysis frequencies $2 \pi k / T, k=1,2, \ldots, N-1$. When the data record length is larger than the reciprocal of the analysis frequency resolution in $\mathrm{Hz}$, then some components could be omitted from the frequency-domain data, which violates Parseval's theorem stating the equivalence of signal energy in the time and frequency domains ${ }^{1,15}$

$$
\frac{1}{N} \sum_{i=0}^{N-1} x^{2}(i) \Delta t=\frac{2}{N} \sum_{k=0}^{M-1} \tilde{x}^{*}(k) \tilde{x}(k) \Delta f
$$

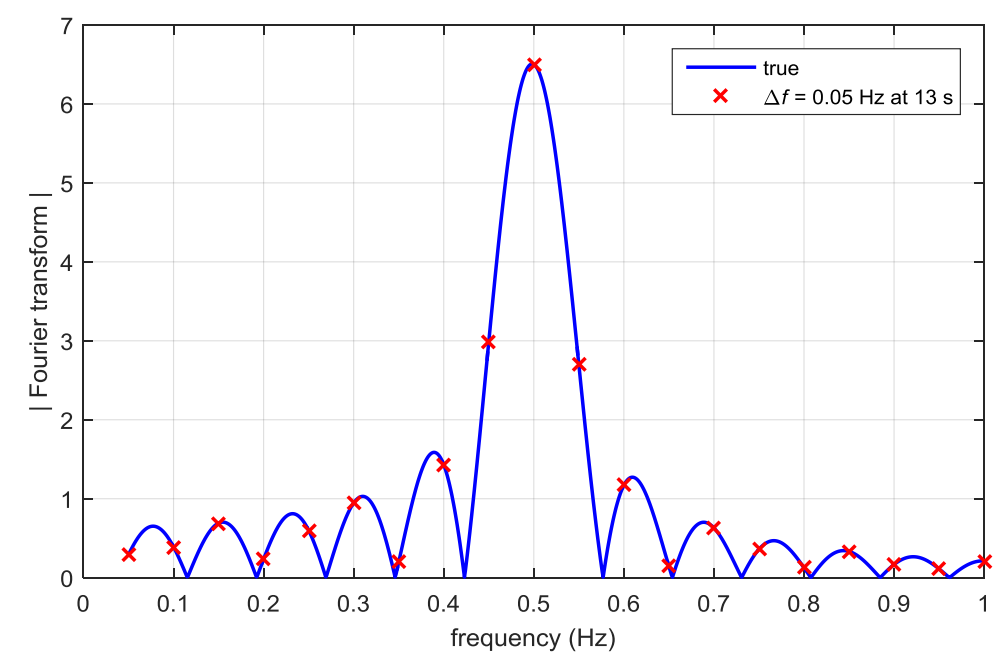

Figure 5. Frequency sampling $\Delta f=1 /(20 \mathrm{~s})=0.05 \mathrm{~Hz}$ for $x(t)=\sin (\pi t)$ at $13 \mathrm{~s}$

As a practical consequence, the analysis frequency resolution must be no larger than $1 / T \mathrm{~Hz}$, where $T$ is the data record length, but the frequency resolution can be arbitrarily finer using the chirp $z$-transform and the highaccuracy calculation of the finite Fourier transform implemented in SIDPAC. Note that some components will also be omitted if the frequency band selected for the finite Fourier transform is smaller than $\left[0, f_{N}\right]$, where $f_{N}$ is the 
Nyquist frequency in $\mathrm{Hz}, f_{N}=f_{s} / 2=1 /(2 \Delta t)$. The omission in the former case is because the frequency resolution is too coarse for the given data record length, whereas in the latter case, the omission is because of a deliberate truncation of the range of analysis frequencies, which can be used for practical advantages, as will be described later.

Figure 6 shows the result from using a very fine and irrational frequency spacing equal to $1 /(3 \pi T) \mathrm{Hz}$. This demonstrates that the analysis frequencies can be made arbitrarily fine without compromising the Fourier transform accuracy when using the high-accuracy Fourier transform in SIDPAC.

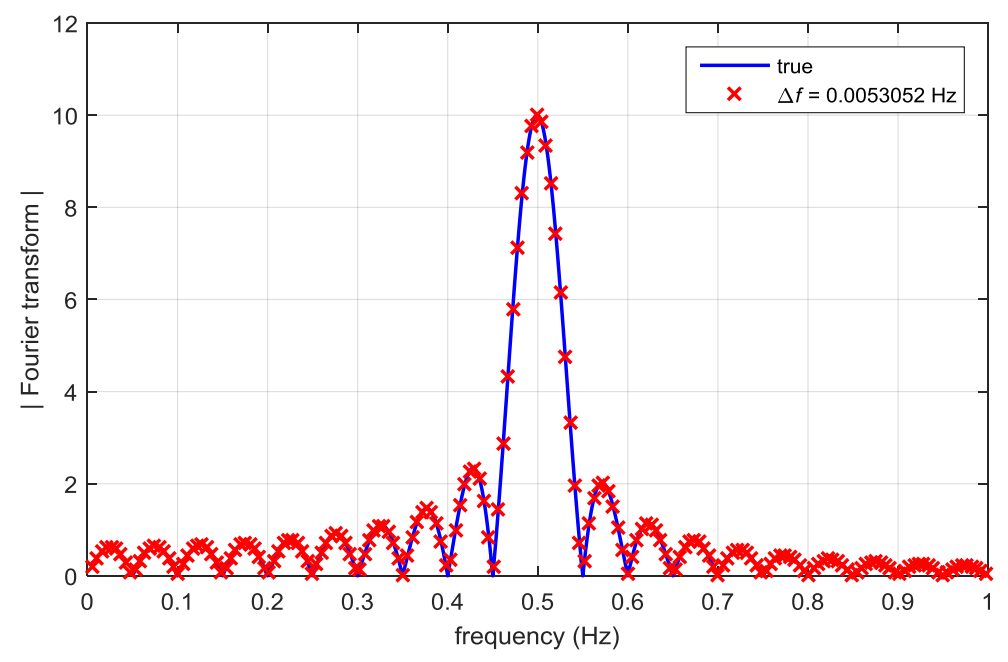

Figure 6. High resolution frequency sampling $\Delta f=1 /(3 \pi 20 \mathrm{~s})=0.0053052 \mathrm{~Hz}$ for $x(t)=\sin (\pi t)$ at $20 \mathrm{~s}$

\section{B. Detrending}

Prior to transforming time-domain data to the frequency domain, any low-frequency signal components with relatively high amplitude, such as the constant part (called the bias) and part that is linearly related to time (called the linear trend or drift), are removed from the time series. This process is called detrending. The purpose of detrending is to prevent leakage from these relatively large low-frequency components, which can pollute the frequency-domain data at the lower end of the frequency range of interest.

Figure 7 shows the effect of leakage using an angle of attack measurement from the T-2 aircraft. The plots on the left side show the measured angle of attack and the corresponding finite Fourier transform data. On the right side, the bias and linear trend were removed from the angle of attack time series prior to applying the finite Fourier transform. The results show that detrending the time series removes the leakage from the relatively large bias and linear trend, and clearly reveals the underlying character of the data at low frequencies. For this reason, detrending is applied before any Fourier transform is applied in practice.

\section{Bias and Trend Removal}

An effective way to detrend a measured time series is to use simple regression to remove the components that are constant and linearly related to time. For a vector $z$ of measured time series data,

$$
\begin{gathered}
\boldsymbol{\theta}_{d}=\left[\begin{array}{ll}
\theta_{0} & \theta_{1}
\end{array}\right]^{T} \quad \boldsymbol{X}_{d}=\left[\begin{array}{ll}
\mathbf{1} & \boldsymbol{t}
\end{array}\right] \\
\hat{\boldsymbol{\theta}}_{d}=\left(\boldsymbol{X}_{d}^{T} \boldsymbol{X}_{d}\right)^{-1} \boldsymbol{X}_{d}^{T} \boldsymbol{z}
\end{gathered}
$$

so that the trend is

$$
\boldsymbol{y}_{d}=\boldsymbol{X}_{d} \boldsymbol{\theta}_{d}
$$



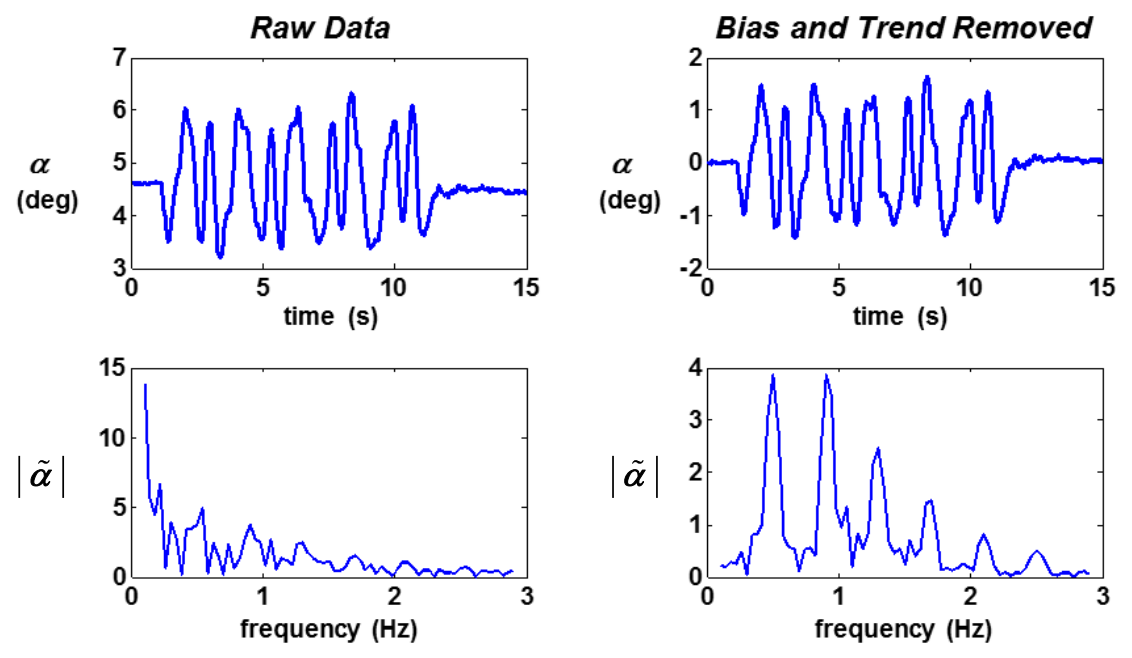

Figure 7. Frequency-domain effect of detrending

The detrended data $z_{d}$ is then

$$
z_{d}=\boldsymbol{z}-\boldsymbol{y}_{d}
$$

Because time series are detrended prior to Fourier transformation, the bias and trend are not observable using frequency-domain modeling. Stated another way, frequency-domain data include only dynamic information content, and not static or trend information. Consequently, for data analysis and modeling in the frequency domain, a separate subsequent step must be included to determine the model bias and trend. Fortunately, this step is easy to accomplish in the time domain, because these terms are typically among the most well-conditioned of all model terms in the time domain, and therefore are easy to estimate accurately.

Removing the linear trend for a data record with length $T$ seconds can be viewed as approximately removing the frequency content of a sinusoid with period $4 T$ seconds. The trend can be considered the first quarter of a triangular wave, approximating a sine wave with the same period. This viewpoint is useful for estimating the effective frequency content being removed when detrending the data.

\section{Nonlinear Detrending}

In some cases, measured time series have large low-frequency components that are more complex than just a bias and linear trend with time. This happens in operational flight maneuvers and in flight test maneuvers designed for global aerodynamic modeling ${ }^{20,21}$. The situation can be handled by augmenting the detrending functions to include higher powers of the time vector. Modifying Eqs. (16a) to include square and cubic terms,

$$
\boldsymbol{\theta}_{d}=\left[\begin{array}{llll}
\theta_{0} & \theta_{1} & \theta_{2} & \theta_{3}
\end{array}\right]^{T} \quad \boldsymbol{X}_{d}=\left[\begin{array}{llll}
\mathbf{1} & \boldsymbol{t} & \boldsymbol{t}^{2} & \boldsymbol{t}^{3}
\end{array}\right]
$$

Eqs. (16b)-(18) can be applied as before. This approach expands the range of flight maneuvers that can be analyzed in the frequency domain. In this case, the frequency content of the detrended components $\boldsymbol{y}_{d}$ cannot be as easily estimated as when only the bias and linear trend are removed, but the trend $\boldsymbol{y}_{d}$ can be analyzed separately to determine the frequency content of the components removed by detrending. With more capable modeling functions being used for detrending, there is a risk of inadvertently removing higher-frequency content, so nonlinear detrending must be done carefully, and the frequency content of the trend $\boldsymbol{y}_{d}$ should be examined.

\section{High-Pass Filtering}

Low-frequency components of a measured time series can be removed using a high-pass filter to implement detrending. The advantage of this approach is that it can be used in real time as well as post-flight. However, the frequency cutoff for any high-pass filter design is gradual, which means part of the large, low-frequency components can get through, and there is some phase lag associated with high-pass filtering near the filter cutoff frequency. These problems can be mitigated with careful design of the high-pass filter, but cannot be avoided completely. Desirable characteristics for the high-pass filter are: sharp frequency cutoff between the frequencies 
passed through and filtered (generally associated with higher-order filters), and small amplitude and phase changes for frequencies in the pass band. Good results have been obtained using a $4^{\text {th }}$-order Butterworth high-pass filter with cutoff frequency set at $1 /(2 T) \mathrm{Hz}$, where $T$ is the period of the lowest frequency of interest. Of course, other highpass filter design solutions are possible. This approach has been applied successfully in flight for real-time dynamic modeling in the frequency domain ${ }^{5,20,22}$.

\section{Automatic Smoothing and Dropout Rejection}

Wideband measurement noise and occasional data dropouts appear in the finite Fourier transform data as relatively constant magnitude components with random phase angles over the entire available frequency range $\left[0, f_{N}\right] \mathrm{Hz}^{23-25}$. A simple way to effectively smooth the data and remove occasional data dropouts is to confine the frequency band used for the finite Fourier transform to a small frequency band associated with the dynamics of interest. In a sense, this is getting something for nothing, because omitting the finite Fourier transform calculation for frequencies outside the band of interest implements data smoothing and occasional data dropout removal.

This is demonstrated in Fig. 8, which shows noisy measured time series data with a single data dropout in the upper left plot. The plots on the left side of Fig. 8 show time and frequency domain data for the measured time series, and the plots on the right show corresponding plots for the smoothed result from applying the finite Fourier transform using the limited frequency band $[0,2] \mathrm{Hz}$ with frequency resolution $\Delta f=0.05 \mathrm{~Hz}$.
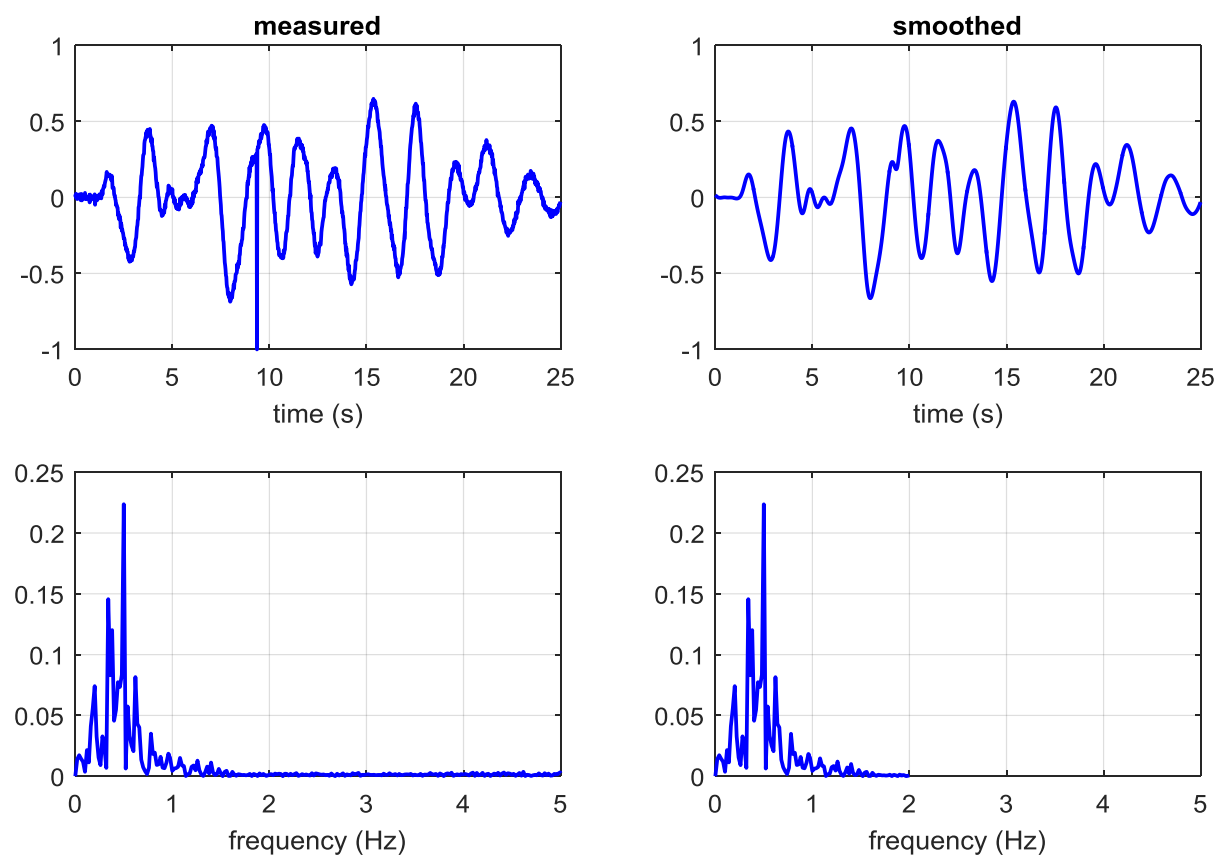

Figure 8. Data smoothing and data dropout removal using Fourier transformation with a limited frequency band

Note that the transformation from the frequency domain back to the time domain (cf. Eq. (8b), result illustrated in the upper right plot of Fig. 8) need not be done when the analysis and modeling are done in the frequency domain. The smoothing is implemented in the frequency domain simply by applying the finite Fourier transform with a limited frequency band, which can be done easily with SIDPAC program czts.m or fint.m. This operation can also be understood from Eq. (2) as a projection of the noisy signal with data dropouts onto relatively low-frequency sinusoids, which cannot represent rapidly-changing time-domain features, so the noise and data dropouts are effectively rejected. When the magnitude of a data dropout is very high, or the noise level is high, then the smoothed data will show some evidence of the undesired signal components, because the high magnitude of the wideband noise or dropout data result in non-negligible contributions within the frequency band of interest. 


\section{Finite Fourier Transform for the Time Derivative of Time Series Data}

When a time series has non-zero endpoints, the finite Fourier transform of the time derivative of that time series requires endpoint correction terms. Applying integration by parts to Eq. (2) for a time derivative $\dot{x}(t)$,

$$
\begin{gathered}
\mathcal{F}[\dot{x}(t)] \equiv \int_{0}^{T} \dot{x}(t) e^{-j \omega t} d t=x(T) e^{-j \omega T}-x(0)+j \omega \int_{0}^{T} x(t) e^{-j \omega t} d t \\
\mathcal{F}[\dot{x}(t)]=x(T) e^{-j \omega T}-x(0)+j \omega \tilde{x}(\omega)
\end{gathered}
$$

Applying high-pass filtering or detrending to a time series or assuming perturbations from an initial reference condition imposes a zero initial condition. This leaves only one term for the endpoint corrections, so that

$$
\mathcal{F}[\dot{x}(t)]=x(T) e^{-j \omega T}+j \omega \tilde{x}(\omega)
$$

Equation (20) can be used to compute the finite Fourier transform for the time derivative of a time series with non-zero endpoints.

\section{E. Finite Fourier Transform for Data from Multiple Maneuvers}

Often the data from more than one maneuver or data record must be combined for a single analysis. When applying the finite Fourier transform, this can be done by simply adding the Fourier transform data from individual data records at corresponding frequencies. Each data record to be combined must be transformed into the frequency domain using the same frequencies. This is easily done when the frequencies for the transformation can be selected arbitrarily, as in the SIDPAC code fint.m. This simple approach works because the finite Fourier transform is an integral.

If frequency-domain data from two maneuvers are to be combined to represent the situation where the associated time series are concatenated in time, the task can be done in the frequency domain by multiplying the frequency domain data from the second maneuver by $e^{-j \omega T_{1}}$, where $T_{1}$ is the time length of the first maneuver, then adding the frequency-domain data at corresponding analysis frequencies $\omega$. This is equivalent to a pure time-shift of the data in the second maneuver by $T_{1}$ seconds.

It is not correct to concatenate data in the time domain, then apply the finite Fourier transform. The reason is that any variations near the boundary of the concatenation but on different sides of that boundary will be erroneously interpreted as real frequency content. For a similar reason, it is not correct to use the total duration of the combined maneuvers for $1 / T$ in frequency-domain analysis. The correct approach is to transform individual maneuvers separately using the same analysis frequencies, then add the frequency-domain data at the corresponding frequencies.

\section{F. Recursive Discrete Fourier transform}

For any given frequency $\omega$, the discrete Fourier transform at sample time $i \Delta t$ is related to the discrete Fourier transform at time $(i-1) \Delta t$ by

$$
X_{i}(\omega)=X_{i-1}(\omega)+x(i) e^{-j \omega i \Delta t}
$$

where

$$
e^{-j \omega i \Delta t}=e^{-j \omega \Delta t} e^{-j \omega(i-1) \Delta t}
$$

This is simply calculating the discrete Fourier transform in Eq. (8a) as a running sum. The quantity $e^{-j \omega \Delta t}$ is constant for a given frequency $\omega$ and constant sampling interval $\Delta t$. It follows that the discrete Fourier transform can be computed for a given frequency at each time step using one addition in Eq. (22a) and two multiplications -

one in Eq. (22b) using the stored constant $e^{-j \omega \Delta t}$ for frequency $\omega$, and one in Eq. (22a). There is no need to store the time-domain data in memory when computing the discrete Fourier transform in this way, because each sampled 
data point is processed immediately as a projection onto Fourier basis functions. Previous time series data can be incorporated in all subsequent analysis by simply continuing the recursive calculation of the Fourier transform. In this sense, the recursive Fourier transform acts as memory for the information in the data. More data from more maneuvers improves the quality of the data in the frequency domain without increasing memory requirements to store it. Furthermore, the Fourier transform is available at any time $i \Delta t$. The approximation to the finite Fourier transform can be completed using Eq. (4b),

$$
\tilde{x}\left(\omega, t_{i}\right) \approx \Delta t X_{i}(\omega)
$$

Figure 9 shows the time evolution of the recursive discrete Fourier transform for frequencies in the range $[0.05,1.0] \mathrm{Hz}$, using the analytic solution in Eq. (13) and the same example sinusoid used earlier, $x(t)=\sin (\pi t)$. Note that the main lobe amplitude increases as the data record length increases, and the side lobes become more numerous and closely-spaced in frequency as time goes on.

The true character of the frequency-domain data is shown in Fig. 9, because the analytic solution for the finite Fourier transform in Eq. (13) was used. Even for this simple sinusoid, it is clear why there might be difficulty in determining power spectral density, because the distribution of signal power with frequency is scattered into side lobes that change significantly as a function of data record length. On the other hand, this changing character is sampled very accurately using the recursive discrete Fourier transform (cf. Figs. 4-6), so that results from the recursive discrete Fourier transform can be used directly at any time as accurate frequency-domain data. This means that any frequency-domain analysis based on discrete Fourier transform data can be readily converted to a real-time method, because of the simple and efficient recursive finite Fourier transform in Eqs. (22). Important practical implications of this will be explored in later sections.

The recursive discrete Fourier transform has been applied successfully in flight tests and in wind-tunnel tests for real-time dynamic modeling in the frequency domain ${ }^{5,20,22,26-33}$.

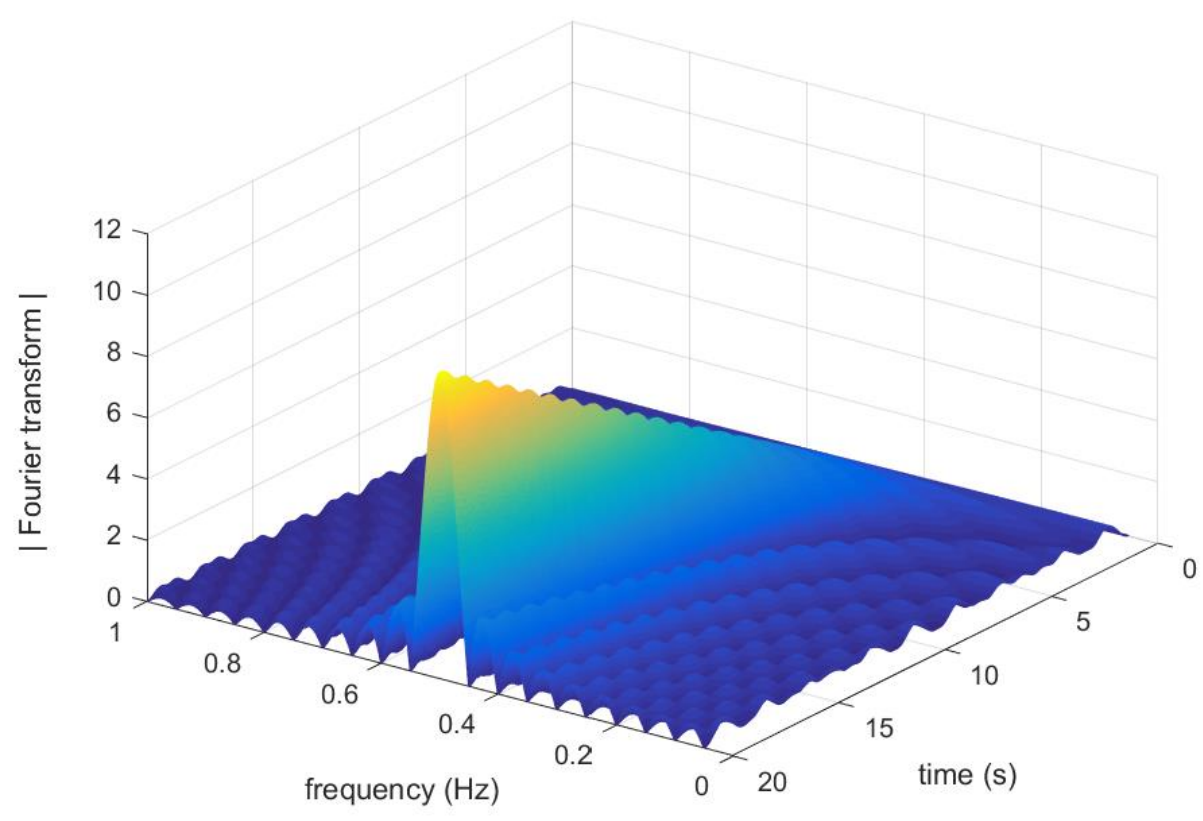

Figure 9. Recursive discrete Fourier transform time evolution

\section{G. Time Variation and Data Forgetting}

The recursive discrete Fourier transform in Eqs. (22) implements a data information memory for as long as the running sum is continued. When the situation changes, older data should be discounted or forgotten in some way. If this is not done, then the speed of response to the changes is progressively degraded, as new information has to overwhelm an increasingly longer memory. Consequently, there is a trade-off between the desired rapid response to changes versus retaining enough data information for accurate data analysis and modeling. 
It is possible to trigger a reduction in the magnitudes of the discrete Fourier transform values computed from past data, based on an event such as a detected failure, a change in test conditions, or a significant increment in the information content of the measured data. In the latter case, the detected increase in recent data information content would presumably support an update to the frequency-domain data. In practice, the criteria and discounting schemes for data forgetting depend on the application.

If past values of the Fourier transform $X_{i}(\omega)$ computed from Eq. (22a) are saved in computer memory, perhaps at set intervals of time, then it is possible to implement selective amnesia by simply subtracting past values of the running sum corresponding to the Fourier transform, or differences between past values of the running sum. For example, forgetting all data information content older than 10 seconds could be implemented by subtracting the value of the running sums for the Fourier transforms at 10 seconds ago from the current running sums. Similarly, to forget data information content collected between 5 and 7 seconds ago, the difference between the running sums at 5 and 7 seconds ago would be subtracted from the current running sum. The price to pay for this capability is the computer memory required to store past values of the running sums associated with the Fourier transforms for each time series at each frequency. The memory requirements could be reduced by perhaps only saving the running sums at intervals of 10 seconds, for example.

The simplicity of Eqs. (22) and linearity of the Fourier transform with respect to the time series data $x(i)$ allows easy implementation exponential data forgetting in the recursive discrete Fourier transform, by modifying Eq. (22a) to

$$
X_{i}(\omega)=\lambda X_{i-1}(\omega)+x(i) e^{-j \omega i \Delta t}
$$

This can be interpreted as multiplying each past value of the time series by a forgetting factor $\lambda, 0<\lambda<1$, at each time step. Old data are gradually devalued and eventually discarded for practical purposes. Use of a constant forgetting factor in this way corresponds to exponential data forgetting with respect to time ${ }^{1}$. Note that $\lambda=1$ makes Eq. (24) the same as Eq. (22a), corresponding to no data forgetting, and $\lambda=0$ would be the impractical case of forgetting all data except the current data point. In practice, typical values of the forgetting factor are

$$
0.90 \leq \lambda<1.00
$$

The challenge is generally not in the implementation of data forgetting, but rather in deciding how much data information to forget, and when. There are currently no concrete guidelines for choosing a value for $\lambda$, nor for determining when and how much to forget using selective amnesia. Alternatively, the recursive discrete Fourier transform can be simply restarted by setting all $X(\omega)$ values to zero based on an event, such as a flight condition change, the start of a new maneuver, a change to the aircraft configuration, or some type of damage or failure.

\section{Frequency Response Estimation}

The use of frequency response data for aircraft system identification involves some important practical considerations related to the calculation and interpretation of frequency response estimates based on measured data.

\section{A. Interpretation of the Frequency Response}

By definition, the frequency response characterizes the linear dynamic relationship between a single input and a single output. Aircraft are nonlinear dynamic systems with multiple inputs and multiple outputs. Because of this mismatch, using frequency responses for aircraft system identification generally requires significant flight test time and other special considerations during flight testing, data analysis, and modeling. One main reason is that accurate frequency response estimation using conventional spectral analysis methods means that only one control can be moved significantly at any time during the flight testing. A frequency response estimated from flight data collected when more than one input is moving will be biased unless corrective steps are applied in the frequency-domain data conditioning ${ }^{6,18,19}$. Furthermore, each frequency response can only characterize a range of flight conditions and dynamic responses where linearity assumptions are valid.

The frequency response is a nonparametric model, and therefore does not require a parametric model structure. The frequency response is equivalent to fitting the system dynamics with a linear, time-invariant, dynamic system model, without specifying the order of the model. Such models have been referred to as describing functions ${ }^{34}$. The frequency response therefore has no inherent capability to model nonlinearity, apart from combining multiple local linear approximations. 
Although the frequency response can be used to predict transient response, it cannot be identified well directly from transient data. The reason is that a frequency response is exhibited most clearly as the steady-state, forced response for a linear, time-invariant system to a single input, after any transients have died out. Consequently, the flight test maneuvers typically used for frequency response estimation are frequency sweeps with slow variation in the input frequency, which generally require more flight test time than other approaches. A more efficient flight test method for frequency response estimation with multiple inputs and multiple outputs will be discussed later.

\section{B. Power Spectral Density Estimation}

The frequency response can be estimated from measured data as the ratio of the estimated cross-spectral density of the output $y$ to input $u$, divided by the auto-spectral density of the input,

$$
\hat{H}(\omega)=\frac{\hat{G}_{u y}(\omega)}{\hat{G}_{u u}(\omega)}
$$

where

$$
\begin{array}{ll}
\hat{G}_{u u}(\omega)=\frac{2}{T} \tilde{u}^{*}(\omega, T) \tilde{u}(\omega, T) & \omega>0 \\
\hat{G}_{u y}(\omega)=\frac{2}{T} \tilde{u}^{*}(\omega, T) \tilde{y}(\omega, T) & \omega>0
\end{array}
$$

Spectral densities are also called power spectral densities or power spectra. The power spectral density values are a function of frequency and represent the squared magnitude of the signal in a frequency bin centered at a particular frequency. Parseval's theorem stated in Eq. (15) shows this in mathematical form, so that the units of auto-spectral density must be

$$
\text { auto-spectral density units }=[\text { signal units }]^{2} /[\text { frequency units }]
$$

and similarly for the cross-spectral density.

\section{Practical Issues}

There are important practical issues associated with the use of the discrete Fourier transform to compute power spectral density. The first is that the number of frequencies used for the Fourier transformation is finite, so that in practice the underlying continuous spectral density function is characterized by discrete values for small frequency bands centered at each frequency $f_{k}$, namely $\left[f_{k}-\Delta f / 2, f_{k}+\Delta f / 2\right]$, where $\Delta f$ is the frequency resolution. The power spectral density estimates are therefore equivalent values for the continuous frequencies contained in each frequency bin centered on $f_{k}$.

Another issue arises because a finite length of data is used to estimate the power spectrum, which leads to the phenomena called leakage, discussed earlier and encountered in Figs. 4-7. This issue is important because the power spectral density quantifies signal power in discrete bins along the frequency axis, so that leakage causes inaccuracy in power spectral density estimates.

A finite length of data on the time interval $[0, T]$ can be obtained (conceptually) by multiplying an infinite length of data by a function that equals 1 on the time interval $[0, T]$ and is zero otherwise. This is called the boxcar function, shown in the upper plot of Fig. 10. It is well-known that the Fourier transform of the product of two time-domain functions is equivalent to convolution of their Fourier transforms in the frequency domain ${ }^{1,6,18,19}$. The Fourier transform of the boxcar function is fairly wide, with a relatively poor rate of decrease for the side lobes as a function of frequency, as shown in the lower plot of Fig. 10. This behavior is related to the fact that it is difficult for a Fourier series to represent a function with discontinuities, such as the boxcar function. Therefore, the Fourier transform of a finite length of data is equivalent to convolution in the frequency domain of the Fourier transform of the time function of interest (assumed infinite in length) with the Fourier transform of the boxcar function. The result is a smearing or leakage of frequency components from each frequency bin into adjacent ones, resulting in reduced accuracy of the power spectral estimates. Note that there is no leakage if the only frequencies present in the data are those associated with the frequency bins, see the lower plot of Fig. 10. 
To mitigate leakage, the finite data record can be multiplied by a windowing function that changes gradually from zero to one, then returns to zero. A windowing function without the severe discontinuity of the boxcar function has fewer and smaller side lobes, which reduces leakage. There are many windowing functions that can be used $^{6,15,18,19}$. A good practical choice is the Bartlett window, defined by

$$
w(i)=1-\left|\frac{i-N / 2}{N / 2}\right| \quad i=0,1, \ldots, N-1
$$

which is a simple ramp from 0 to 1 and back to 0 . When data windowing is implemented, the time series $x(i)$ is modified to $w(i) x(i)$, where $w(i)$ is the value of the windowing function at the $i$ th data point. To maintain the validity of Parseval's theorem, the power spectral density estimates must be divided by the mean sum of squares of the weighting function,

$$
\frac{1}{N} \sum_{i=0}^{N-1} x^{2}(i)=\frac{1}{N \sum_{i=0}^{N-1} w^{2}(i)} \sum_{k=0}^{N-1} X_{w}(k) X_{w}^{*}(k)
$$

where $X_{w}(k)$ is computed from the windowed time series $w(i) x(i), i=0,1, \ldots, N-1$.
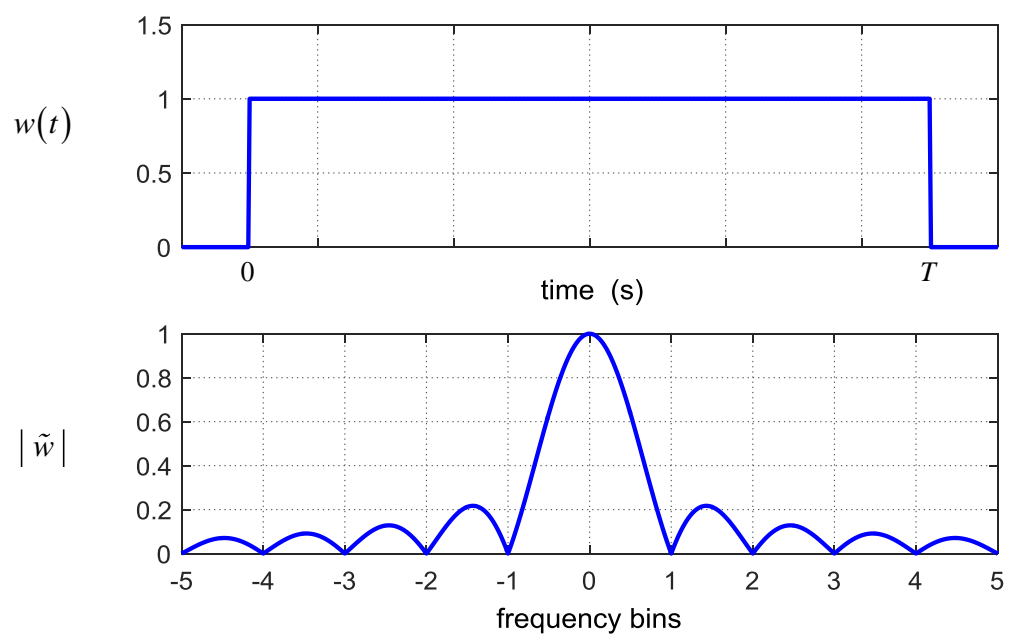

Figure 10. Boxcar function and its Fourier transform

Another important issue relates to the accuracy of power spectral estimates computed from measured time series data using Eqs. (27). Spectral density estimates computed directly from Eqs. (27) have random error close to 100\% of the computed values, regardless of the data record length ${ }^{15,18}$. In practice, this random error can be reduced using some form of averaging. One common method is to partition the measured time series into $n$ segments of data. The standard calculation of the spectral densities in Eqs. (27) is applied to each data segment. Then the results at each frequency are averaged, resulting in a spectral density estimate with random error variance reduced by a factor of $1 / n$, due to the averaging. To achieve more averaging, the data segments are normally overlapped by $50 \%$, meaning that adjacent segments share $50 \%$ of their data points. This increases the number of averages $n$, but decreases the variance reduction factor to $9 /(11 n)$, because some data are reused in adjacent data segments. However, the increased number of averages from overlapping data segments more than compensates for the decrease in the variance reduction factor.

However, partitioning splits the data into subsets with smaller data record lengths, and this raises the lowest frequency available and makes the frequency resolution more coarse. Although overlapping the data partitions by up to 50 percent of the window width mitigates the problem, the fundamental trade-off between reduced random error (for an increased number of smaller windows) and reduced frequency range and resolution (for fewer larger 
windows) remains. This problem has been addressed by analyzing the data using various window sizes, then combining the results using an optimized weighting based on the extent to which the output is linearly related to the input, quantified by coherence ${ }^{6}$

$$
\gamma_{u y}^{2}(\omega)=\frac{\left|G_{u y}(\omega)\right|^{2}}{G_{u u}(\omega) G_{y y}(\omega)} \quad 0 \leq \gamma_{u y}^{2}(\omega) \leq 1
$$

A coherence value of 1 represents a perfectly linear relationship between the input $u$ and output $y$, whereas a coherence value of 0 means that the input and output have no linear relationship. The coherence is a function of frequency.

Another way to address the problem is to simply increase the maneuver time length, but that approach requires more flight test time and can be impractical for flight conditions that cannot be maintained for a long period of time.

Note that the approach of windowing and averaging has an implicit assumption that the windows of data are different samples of a stationary random process assumed to have properties that are constant with time. If the data come from an aircraft excited using a conventional frequency sweep, then the frequencies change sequentially in time. In that case, the stationarity assumption is not valid because the data for each window has different frequency content, and the accuracy of the spectral estimates computed using the windowing method is compromised. Figure 11 illustrates the idea for a simulated longitudinal flight test maneuver with a frequency sweep input applied to the longitudinal pilot input $\eta$, showing Bartlett data windows with 50 percent overlap.
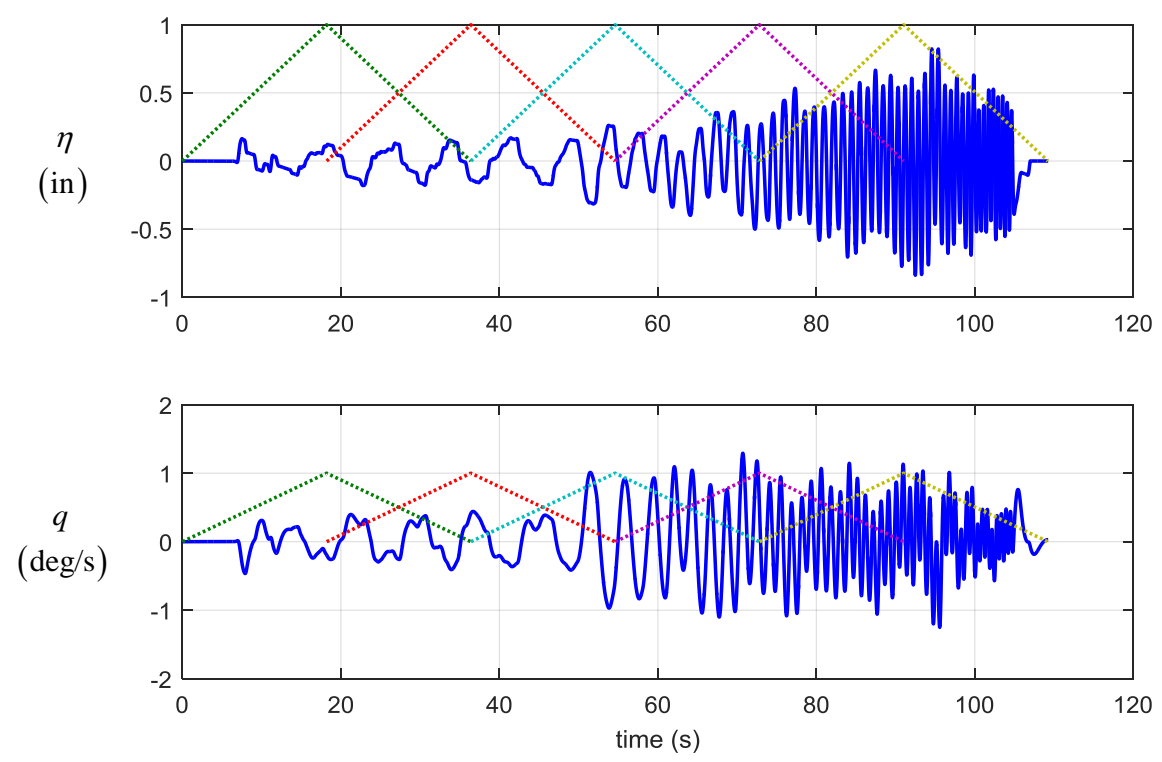

Figure 11. Longitudinal frequency sweep data and data windows

An alternative method for reducing the variance in the spectral density estimates is to compute the Fourier transform at a frequency resolution $n$ times finer than is desired for the end result. SIDPAC routines czts.m or fint.m can be used to compute the spectral densities on the fine frequency mesh, then the results are summed for adjacent $n$ frequencies to produce results for the desired (coarser) frequency mesh. This results in spectral density estimates with random error variance reduced by a factor of $1 / n$. The spectral density values are summed rather than averaged, to maintain the validity of Parseval's theorem. This summing operation is also consistent with the earlier discussion concerning discrete frequency bins.

The binning method is easy to implement, because the chirp $z$-transform implemented in SIDPAC codes czts.m or fint.m has the capability for arbitrary frequency resolution. The approach is simply to determine the number of values $n$ required to achieve the desired accuracy for the spectral density estimates, and select a frequency mesh fine enough to provide the required number of adjacent values. Then implement Eqs. (27) and sum the adjacent 
results to compute an accurate spectral estimate at each desired frequency. This method is implemented in SIDPAC programs fresp.m, spect.m, and cspect.m.

For long data records, frequency responses can be estimated accurately by averaging results from overlapped windowed data segments, sometimes called the periodogram method, and from the binning method. An enhancement of the periodogram method is implemented in the commercial software called CIFER ${ }^{\circledR 6}$, where periodogram estimates using windows with various sizes are combined using an optimization based on coherence to improve the estimates of power spectra and frequency responses. Figure 12 shows frequency response estimates from these three methods, using the frequency sweep data shown in Fig. 11. The magnitude and phase plots for a $q / \eta$ longitudinal frequency response are shown on the left side of Fig. 12. The data shown in Fig. 11 were generated using a known simulation, so that the true dynamic system was known. The true frequency response is plotted using solid lines on the left side of Fig. 12. The errors between the frequency response estimates and the true frequency response are shown on the right side of Fig. 12 for both magnitude and phase angle. In this case, the accuracy of the three methods is comparable.
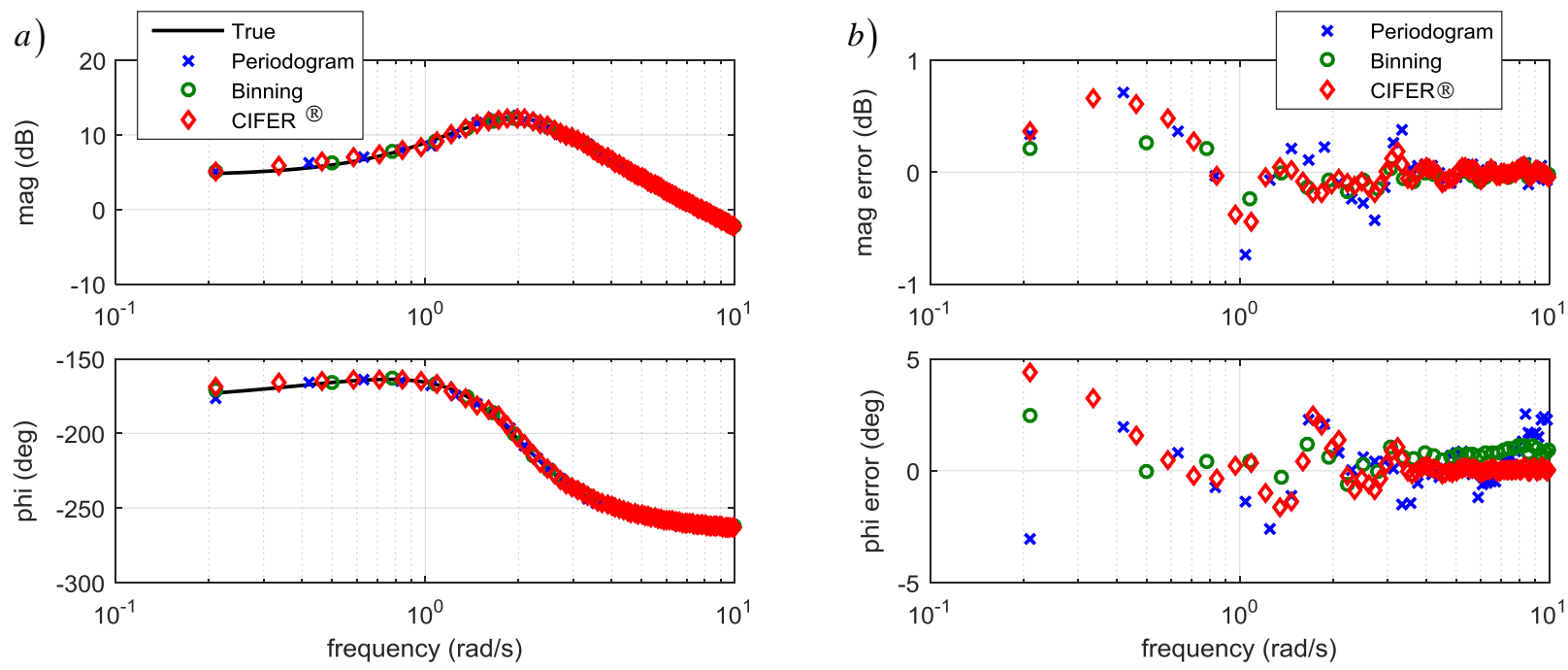

Figure 12. Longitudinal $q / \eta$ frequency response, $a$ ) estimates and $b$ ) errors

Next, the simulation model was modified so that the damping was lower, and the data record length was reduced to 20 seconds, as shown in Fig. 13. A computer-generated frequency sweep was used for the longitudinal input $\eta$ over the frequency range $[0.63,10.0] \mathrm{rad} / \mathrm{s}$ or $[0.10,1.59] \mathrm{Hz}$.

In this case, the periodogram method was applied using a window length of $4 \mathrm{~s}$, which leads to 9 data windows overlapping by 50 percent. Algorithm settings were adjusted so that the periodogram method and the binning method produced frequency response estimates at approximately the same frequencies.

Frequency response estimation results are shown in Fig. 14. The periodogram method produced relatively poor results at middle and lower frequencies. Furthermore, the values produced at resolutions higher than the inverse of the window length in seconds $(1 / 4 \mathrm{~Hz}$ in this case) are actually interpolations. The lightly damped dynamics and relatively short data record were chosen to highlight this issue. On the other hand, the binning approach does not interpolate, but rather produces accurate estimates of the true frequency response based on high-quality Fourier transform data with very fine frequency resolution. The binning method does not have degraded accuracy at middle and lower frequencies, which the periodogram has because there are fewer cycles of the lower frequencies in each data window. The binning method does not window the data, but instead uses the entire data record, because the averaging for improved accuracy is done by summing high-quality Fourier transform data with very fine resolution.

Any method based on the periodogram has an inherent trade-off between the data record length needed for a sufficient number of data windows for averaging to reduce random error, and the frequency resolution, which is $1 / T_{w}$, where $T_{w}$ is the length of the data window in seconds. The binning method avoids this problem by using high-resolution, high-accuracy Fourier transform data for the entire data record, and summing the high resolution data to get accurate frequency response estimates. Consequently, for short data records, the binning method can provide more accurate results at higher frequency resolutions compared to methods based on the periodogram. 

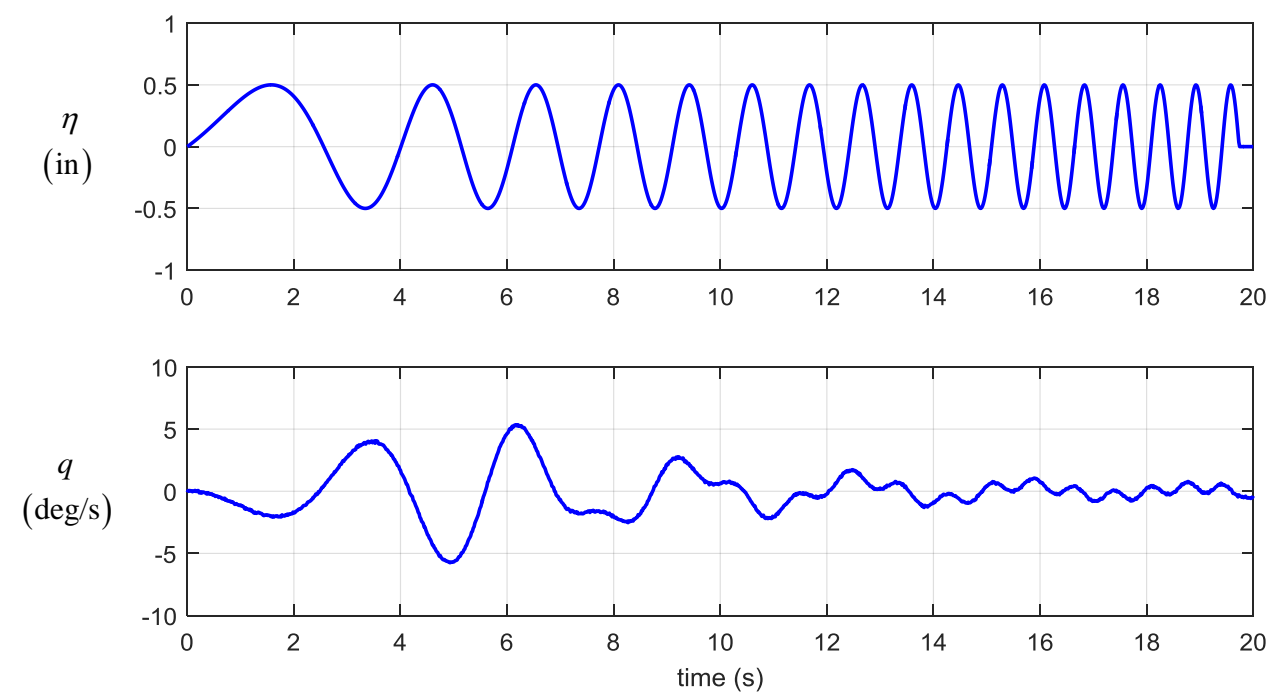

Figure 13. Longitudinal frequency sweep data
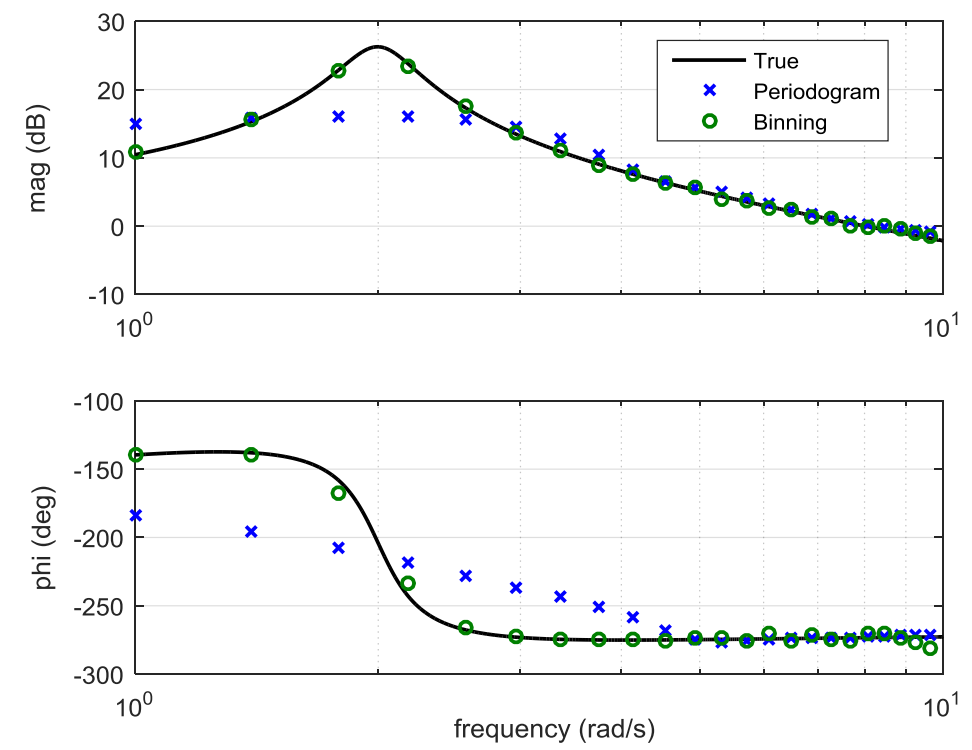

Figure 14. Longitudinal $q / \eta$ frequency response estimates for a $20 \mathrm{~s}$ frequency sweep

\section{Removing Endpoint Discontinuities for Accurate Spectral Analysis}

Fourier analysis implicitly assumes that the time series under consideration is periodic. For most measured time series, making such an assumption implies discontinuities in the amplitude and first time derivative at the endpoints. This is illustrated in the upper plot of Fig. 15, which shows a time series constructed with known frequency content, but made very similar to a measured time series for sideslip angle from a T-2 flight test maneuver. Lanczos ${ }^{23}$ showed that the Fourier series for time series with these endpoint discontinuities has much slower convergence than the Fourier series for a function with no endpoint discontinuities in either the amplitude or first time derivative. In the former case, the magnitudes of the Fourier coefficients decrease asymptotically with $k^{-1}$, where $k$ is the number of terms in the Fourier series expansion, whereas in the latter case, the asymptotic decrease goes according to $k^{-3}$. This seems reasonable, because the sinusoids in the Fourier series have no discontinuities in amplitude or 
first derivative anywhere, and therefore would be expected to have difficulty representing a time series with those discontinuities.

Note that endpoint discontinuities typically exist even after detrending is applied, mainly because amplitude variations in practical time series are seldom symmetric about any removed trend. The lower plot in Fig. 15 shows the same sideslip angle data with bias and linear trend removed. Discontinuities in magnitude and slope at the endpoints remain, although they are not as large as for the original time series shown in the upper plot of Fig. 15. Consequently, even a detrended time series can have degraded Fourier series convergence, although usually to a lesser extent than if the time series were not detrended. There are times when removing only the bias and linear trend results in worse discontinuities at the endpoints than the original time series. This usually happens when the time series has a non-zero mean or linear trend with time, or the low-frequency time variation is something more complicated than linear with time.

It is possible to remove the endpoint discontinuities from any arbitrary time series, and thereby achieve the more abrupt decrease in the magnitude of the Fourier coefficients, which corresponds to faster convergence of the Fourier series expansion. The higher rate of convergence can be used to enable separation of deterministic signal from random noise ${ }^{1,24}$, but also for accurate spectral analysis.
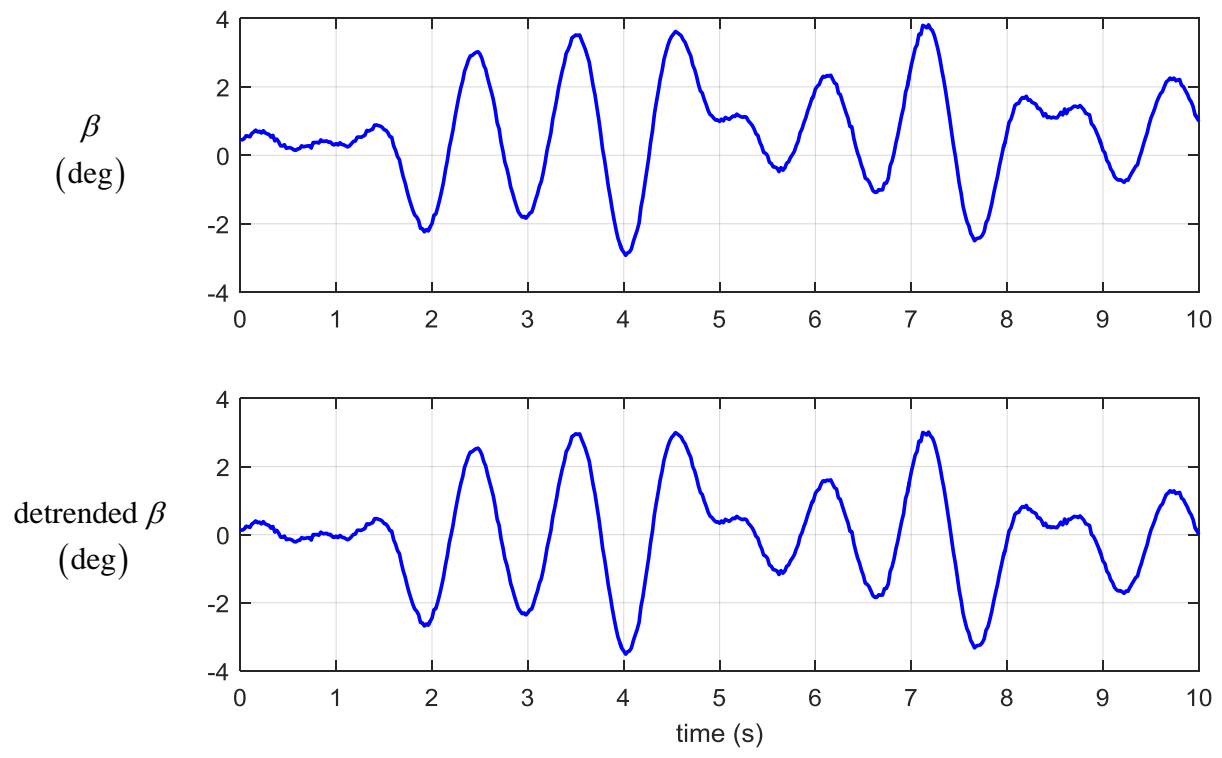

Figure 15. Sideslip angle time series

To remove the discontinuities, a linear trend is subtracted from measured time series $\boldsymbol{x}$ to pin the endpoints to zero, then the result is reflected about the origin to remove both the magnitude and slope discontinuities at the endpoints. In practice, the endpoint data used to do this are locally smoothed values (several SIDPAC tools can be used for this, e.g., Ismep.m), so that the effect of noisy endpoints on the removed linear trend is minimized.

Then define a new time series $g(i)$, with $g(-N+1)=g(0)=g(N-1)=0$, computed as

$$
\begin{array}{cc}
g(i)=x(i)-x(0)-i\left[\frac{x(N-1)-x(0)}{N-1}\right] & i=1,2, \ldots, N-1 \\
g(-i)=-g(i) & i=1,2, \ldots, N-1
\end{array}
$$

Figure 16 shows the result of performing these operations on the time series shown in the upper plot of Fig. 15. The vector

$$
\boldsymbol{g}=\left[\begin{array}{llllllll}
g(-N+1) & g(-N+2) & \ldots & g(-1) & g(0) & g(1) & \ldots & g(N-1)
\end{array}\right]^{T}
$$


is an odd function of time, and therefore can be expanded using a Fourier sine series:

$$
\hat{g}(i)=\sum_{k=1}^{N-1} b_{k} \sin \left[k \pi\left(\frac{i}{N-1}\right)\right] \quad i=1,2, \ldots, N-1
$$

where $\hat{g}(i)$ denotes the approximation to $g(i)$ using the Fourier sine series and $b_{k}$ are Fourier sine series coefficients. The summation over frequency index $k$ omits $k=0$ (zero frequency), since this is a pure sine series for an odd function. Only positive values of $i$ are included in Eq. (33), because those values correspond to the original time series.

The abrupt $k^{-3}$ decrease in the magnitudes of the Fourier sine series coefficients $b_{k}$ can now be expected because the discontinuities in the amplitude and first time derivative at the endpoints have been removed.

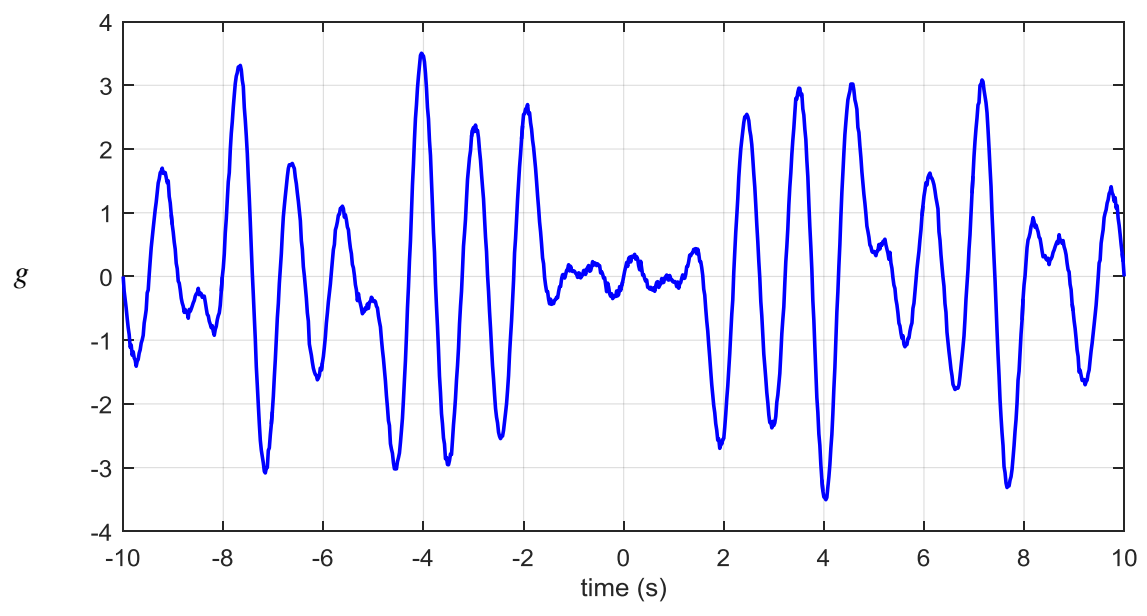

Figure 16. Sideslip angle time series with endpoint discontinuities removed

The Fourier sine series coefficients for $\boldsymbol{g}$ are computed as s.,23,24 $^{1}$

$$
b_{k}=\frac{2}{N-1} \sum_{i=1}^{N-2} g(i) \sin \left[k \pi\left(\frac{i}{N-1}\right)\right] \quad k=1,2, \ldots, N-1
$$

where the index $i$ runs from 1 to $(N-2)$, because $g(0)$ and $g(N-1)$ are zero by Eq. (32a). The upper limit for $k$ is $N-1$, which corresponds to the Nyquist frequency. From Eq. (34), the period $T_{k}$ of the $k$ th Fourier sine series term is given by

$$
T_{k}=\frac{2(N-1) \Delta t}{k}
$$

so the $k$ th frequency $f_{k}$ is related to the frequency index $k$ by

$$
f_{k}=\frac{k}{2(N-1) \Delta t}
$$

Using Eq. (33) to construct $\boldsymbol{g}$ guarantees that any endpoint discontinuities are in the second and higher time derivatives of the time series. Convergence of the Fourier sine series approximation would be further accelerated if endpoint discontinuities in higher derivatives were also removed. However, since the measured time series are from a dynamic system where application of forces and moments can produce discontinuities in the second and higher time derivatives, any attempt to remove additional endpoint discontinuities risks inadvertently modifying the signal. Therefore, removing discontinuities in the amplitude and first time derivative at the endpoints is accepted as the best that can be done without corrupting the desired signal. 
Spectral analysis done in this way is straightforward, general, and accurate. Figure 17 shows spectral estimation results for the time series shown in the top plot of Fig. 15, compared to the known spectrum. Because the technique converts a finite time series with endpoint discontinuities into a time series that is periodic (cf. Fig. 16), the adverse effects arising from convolving the Fourier transform of the time series spectrum with the Fourier transform of the boxcar function are avoided, and the windowing and averaging operations required for conventional spectral analysis are not necessary. In addition to spectral analysis, other practical applications of this approach include noise covariance estimation from measured time series data, zero-lag data smoothing, zero-lag data extraction for arbitrarily selected frequency bands, and random noise generation ${ }^{1,23-25}$.

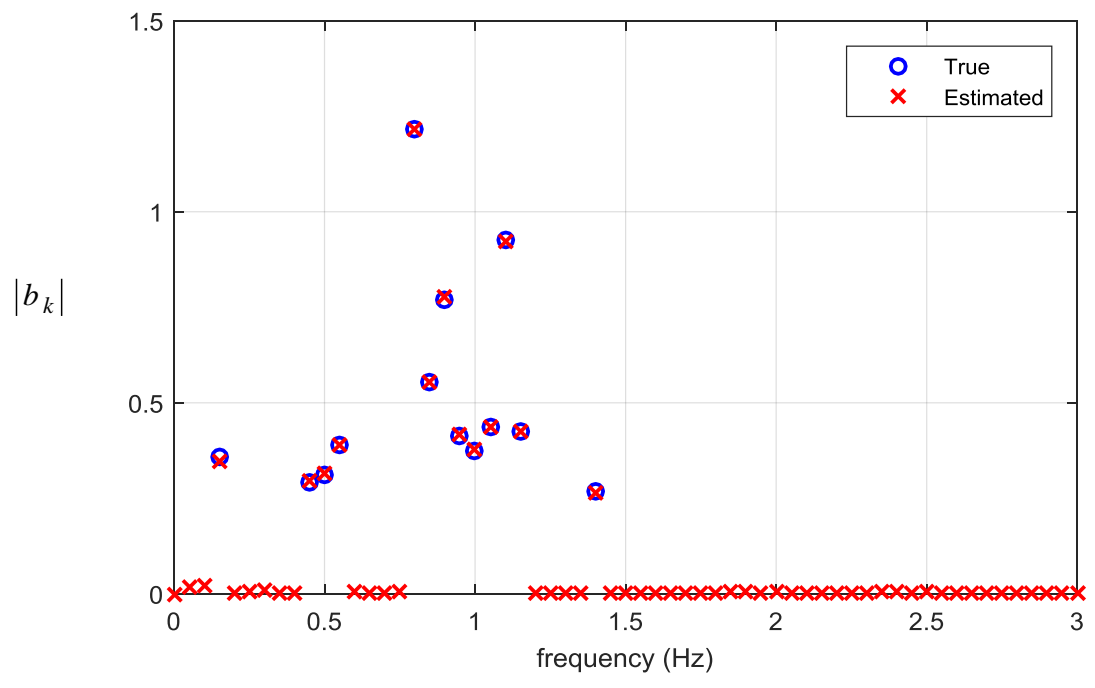

Figure 17. Spectral analysis for sideslip angle time series

\section{Discrete input frequency estimates using orthogonal optimized multisine inputs}

The spectral density estimation expressions in Eqs. (27) are based on the analysis of stationary random data, where the spectral density as a function of frequency is unknown. This knowledge deficiency leads to the uncertainty in the spectral estimates, and drives the need to use averaging techniques to obtain spectral estimates with acceptable accuracy. Such techniques are appropriate when the input has frequency content that is not precisely known prior to the analysis, such as when piloted frequency sweeps are used for aircraft flight testing. Note that frequency responses cannot be calculated accurately for any analysis frequency where input power is low or nonexistent, because that would be close to dividing by zero, cf. Eq. (26).

An alternate approach to frequency response estimation is to use orthogonal optimized multisine inputs ${ }^{1,35,36}$, which have frequency content that is discrete and known prior to the analysis. The spectral content in the output is then known to lie at the designed discrete input frequencies, assuming linear system dynamics, which is implicit in the use of frequency responses. This greatly simplifies the calculation of frequency responses, because frequency response values can be computed directly at the discrete frequencies where input power is known to exist, using the simple expression

$$
\hat{H}(\omega)=\frac{\tilde{y}(\omega)}{\tilde{u}(\omega)}
$$

Error can be introduced because of the nonlinearity inherent in all practical aircraft, which produces spectral content in the output at frequencies other than the input frequencies. However, this error is present in any case when using frequency responses, which characterize only linear relationships.

Orthogonal optimized multisine inputs can be applied to multiple inputs at the same time, so that many frequency responses can be extracted simultaneously, rather than only the frequency responses for one input at a time, as must be done when using frequency sweeps and conventional spectral estimation based on Eqs. (26)-(27). Careful design of the multisines and excitation time length can be used to minimize or eliminate leakage.

Orthogonal optimized multisine inputs can apply excitation at discrete frequencies in parallel for many inputs simultaneously, as opposed to a frequency sweep, which applies excitation at sequential frequencies for one input at 
a time. Consequently, using orthogonal optimized multisine inputs provides advantages in reducing flight test time and improving data quality, particularly at lower frequencies, because the dynamic system reaches steady state for all input frequencies relatively close to the beginning of the maneuver. In contrast, a frequency sweep must be lengthy so that the input frequencies can be traversed slowly, to approach steady state for the frequency content applied sequentially. Using discrete frequencies in the inputs also avoids the spectral estimation problems that require windowing, averaging, and coherence weighting, so that the frequency response can be estimated simply with Eq. (36). However, orthogonal optimized multisine inputs must be implemented by a computer. A pilot can approximate these inputs effectively ${ }^{21}$ for accurate dynamic modeling in the time domain and using the frequency domain methods described later. However, for spectral analysis, the piloted approximation produces continual transient response, which degrades spectral estimation results.

To demonstrate the approach, simulated F-16 longitudinal data were collected using a 20-second optimized multisine input. Because only a single input is used, the frequencies contained in the input can be very closely spaced $^{32}$. Figure 18 shows the optimized multisine input and the noisy pitch rate output data.
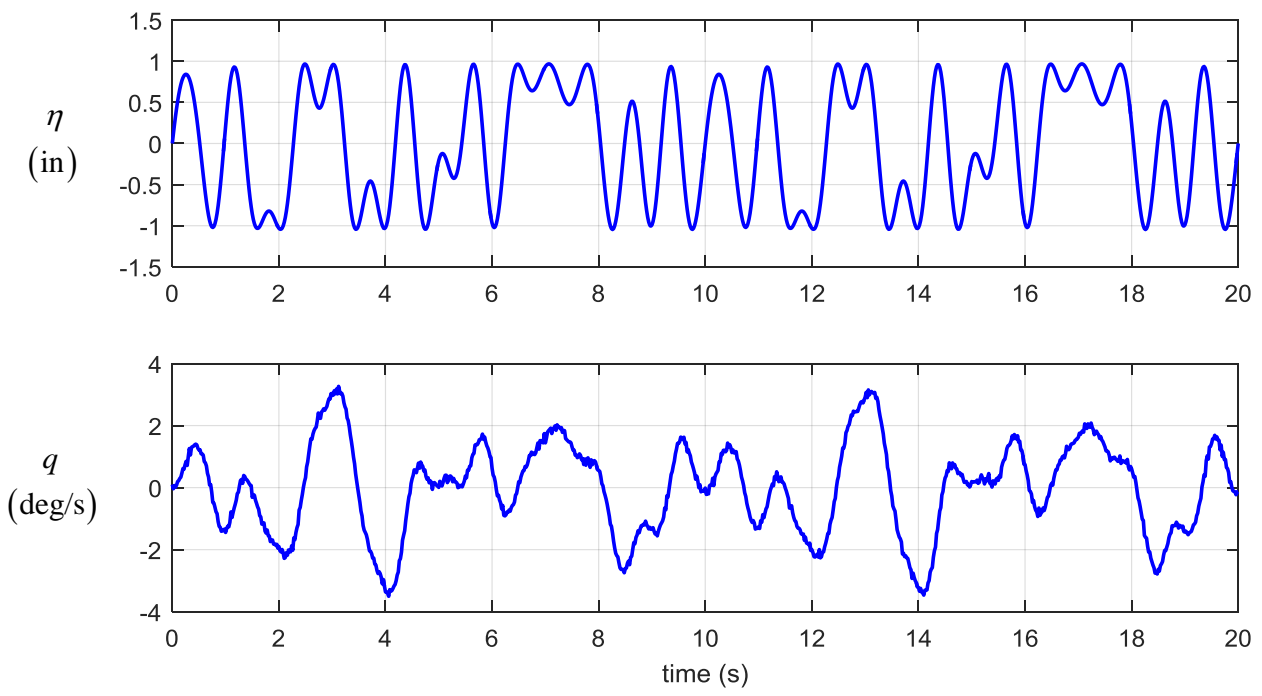

Figure 18. Longitudinal optimized multisine data

Figure 19 shows frequency response estimates at the discrete input frequencies using Eq. (36), along with results using the periodogram method and the binning method, compared to the true frequency response. The discrete frequency estimates are very accurate, and were obtained very simply from Eq. (36). As before when using a conventional frequency sweep input, the binning method produced accurate frequency response results. The periodogram method results are still slightly degraded at middle and low frequencies, but the results are much better when using the optimized multisine input, compared to using a conventional frequency sweep input. This is because the excitation is applied at all frequencies simultaneously from start to finish for the optimized multisine, whereas the excitation is applied serially in frequency for a conventional frequency sweep, making the data information content more complete and dense throughout the maneuver for the optimized multisine input. In addition, the transient dies out early in the maneuver for all frequencies in the optimized multisine input, which improves the data quality for frequency response estimation, whereas the frequency sweep does not have that feature.

For a single input, the frequency content of the optimized multisine, which is also the frequency resolution of the frequency response estimates, can be made finer than $1 / T$, to approximately $1 /(4 T)$ before the differences in the waveforms become too small to be distinguished in the frequency-domain analysis.

The method using discrete input frequencies has the additional advantage that it can be applied without modification to problems with more than one input. Orthogonal optimized multisine inputs can be designed ${ }^{1,35,36}$ and applied simultaneously to the dynamic system, and the frequency response estimation can be done simply and accurately using Eq. (36). The combination of orthogonal optimized multisines and Eq. (36) has been applied successfully in wind tunnel tests with many inputs and outputs ${ }^{29}$, and in flight tests ${ }^{27,28,30-33}$ to efficiently compute frequency responses from time series data. This general approach is also the basis of effective methods for real-time frequency response estimation, both in the time domain ${ }^{27}$, and in the frequency domain ${ }^{28-33}$. 

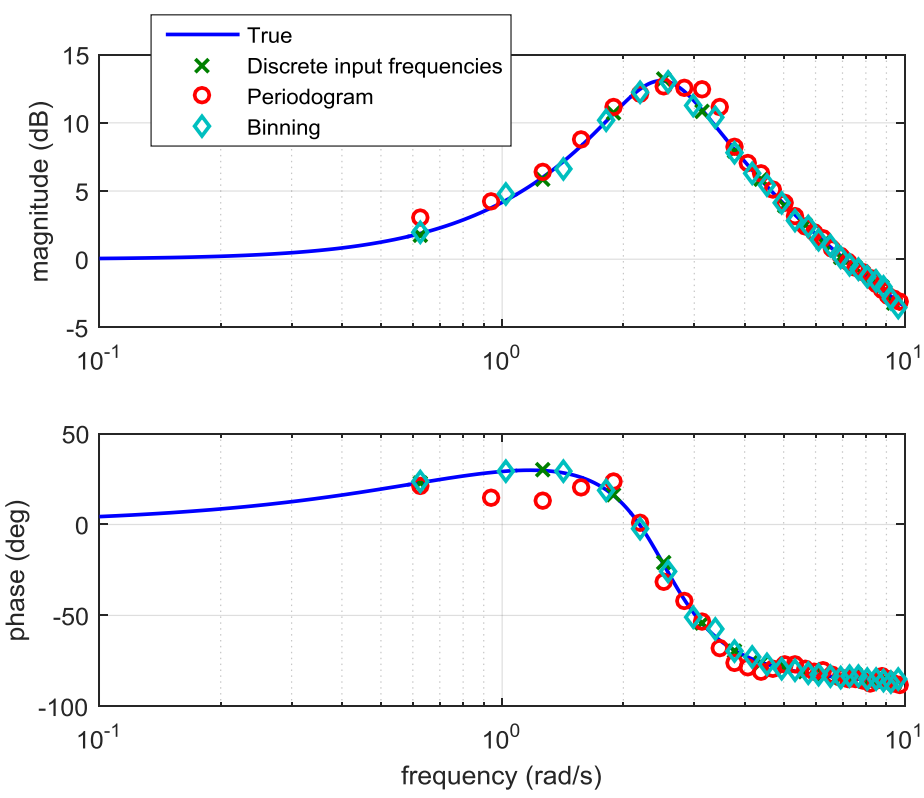

Figure 19. Longitudinal $q / \eta$ frequency response estimates for a 20 s optimized multisine

\section{Effect of transient response on frequency response estimate accuracy}

As discussed earlier, the frequency response characterizes the steady-state response of a linear time-invariant system to sinusoidal inputs or input components. Because estimating frequency responses generally requires steadystate data, any additional content in the data will bias the resulting estimate. Other contributions, such as transient responses, are always present in the data to some extent.

To examine the origin of the transient response for frequency response estimation, consider the solution to the state equation of a linear time-invariant system using Laplace transforms,

$$
\tilde{\boldsymbol{x}}(s)=(s \boldsymbol{I}-\boldsymbol{A})^{-1}\left[\boldsymbol{x}\left(0^{+}\right)+\boldsymbol{B} \tilde{\boldsymbol{u}}(s)\right]
$$

For a single harmonic input $u(t)=\sin \left(\omega_{o} t\right)$, the solution can be expanded using partial fractions as

$$
\tilde{\boldsymbol{x}}(s)=\frac{\boldsymbol{N}_{1}(s) \boldsymbol{x}\left(0^{+}\right)}{\Delta(s)}+\frac{\boldsymbol{N}_{2}(s)}{\Delta(s)}+\frac{\boldsymbol{N}_{3}(s)}{s^{2}+\omega_{o}^{2}}
$$

where $\boldsymbol{\Delta}(s)$ is the characteristic polynomial of the system and the $\boldsymbol{N}(s)$ are matrices with polynomial elements in general. The first term on the right side of Eq. (38) is the free response due to initial conditions, and occurs at the natural frequencies of the system. The second term is the transient response due to offset from the steady-state response, and also occurs at the natural frequencies of the system. The third term is the steady-state linear response, which occurs at the frequency of the harmonic input.

As an example, Fig. 20 shows the Fourier transform of the pitch rate response of the F-16 nonlinear simulation to a sinusoidal input on the stabilator near the short period frequency, for one cycle of the input. A linear model for the short period mode computed from finite differences applied to the nonlinear simulation was used to separate the transient and steady-state responses. In this case, the transient response was a significant portion of the total response, and would impact frequency response estimation results near the short period frequency. 


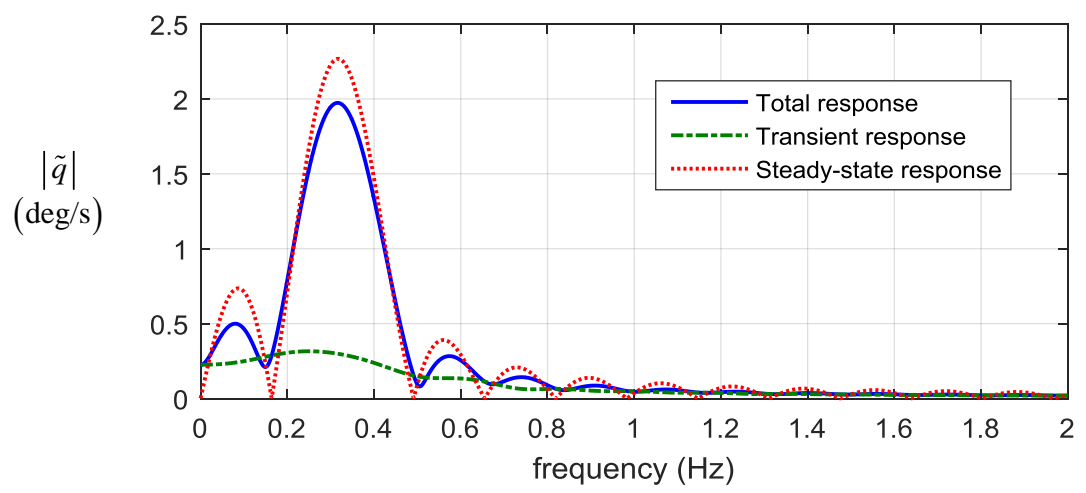

Figure 20. Pitch rate response of the F-16 simulation model to a $0.32 \mathrm{~Hz}$ sinusoidal input

A variety of techniques can be used to mitigate the effects of transient responses in the data. In early experiments for system identification ${ }^{37}$, sine dwells were used, where a single sinusoid was applied, one frequency at a time, for each input. Numerous cycles of data were recorded, but only the last few cycles were averaged to compute relative magnitudes and phases at that single frequency.

A common alternative is to use frequency sweeps, where a harmonic input with increasing frequency is applied to the system. Because the input and steady-state output are continually changing, the second term in Eq. (38) is present throughout the duration of the maneuver. However, if the frequency is increased at a sufficiently slow rate, these transient responses are negligible compared to the steady-state response.

Another option is to use orthogonal optimized multisine inputs. In this context, a multisine input can be viewed as doing numerous sine dwells simultaneously on different control surfaces with different frequencies and phase angle offsets. Transient responses decay early in the data record, and most of the remaining data are entirely the steady-state response. In addition, multiple cycles of the input can be applied to obtain more steady-state data and overwhelm the transient portion of the data, and/or a fraction of the data at the beginning of the maneuver could be discarded. Another alternative is to linearly fade in the input from zero, to reduce the amplitude of the transient response.

\section{Real-Time Frequency Response Estimation}

This section summarizes three techniques developed for real-time estimation of frequency responses from measured flight test data. Conventional frequency response estimation methods ${ }^{6,18,19}$ require the entire data record, after the maneuver has completed. In contrast, these real-time techniques were developed to obtain frequency response estimates during flight tests and to reduce the amount of test time required to meet specified error tolerances. They can also be applied to estimate describing functions for adaptive controllers, time-varying systems for aircraft fault detection, human pilot models, multiple open and closed control loops, and stability margins for expediting envelope expansion flight tests.

Each of these three methods employ multisines on the inputs

$$
u_{j}(t)=\sum_{k \in K} a_{k} \sin \left(\omega_{k} t+\phi_{k}\right)
$$

and assumes the outputs are steady-state responses of the form

$$
y_{i}(t)=\sum_{k \in K} b_{k} \sin \left(\omega_{k} t+\psi_{k}\right)
$$

where $K$ represents the set of all available harmonic frequencies.

The methods recursively estimate either the input and output amplitudes and phase angles, $a_{k}, b_{k}, \phi_{k}$, and $\psi_{k}$, or recursively estimate the input and output Fourier transforms $u_{j}\left(\omega_{k}\right)$ and $y_{i}\left(\omega_{k}\right)$. The corresponding frequency responses are updated in real time as 


$$
\hat{H}_{i j}\left(\omega_{k}\right)=\frac{y_{i}\left(\omega_{k}\right)}{u_{j}\left(\omega_{k}\right)}=\left(\frac{\hat{b}_{k}}{\hat{a}_{k}}\right) e^{j\left(\hat{\psi}_{k}-\hat{\phi}_{k}\right)}
$$

from the $j$ th input to the $i$ th output at the excitation frequencies $\omega_{k}$.

The quality of a frequency response estimate is traditionally judged by its coherence, cf. Eq. (31). Because no windowing or averaging is used in these real-time methods, the coherence is exactly 1 at all frequencies, which does not provide useful information. The analogous metric in this case is the coefficient of determination $R^{2}$, computed for each excitation frequency by comparing component sinusoids in the time domain. In addition, the least-squares approach used in two of the three methods automatically provides uncertainty bounds on the estimated terms, which can be transformed into uncertainties on the Bode plots. Applying a moving average to the time variations of the frequency response estimates provides additional insight.

Figure 21 shows a representative case, illustrated using a Bode plot. The F-16 nonlinear simulation was excited by applying an optimized multisine with 13 frequencies to the stabilator, and pitch rate was used as output. Each blue trace shows the frequency response estimate at one of the excitation frequencies. At the beginning of the maneuver, the estimates vary wildly due to noise, transient responses, and lack of data information. As the excitation continues, the estimates quickly converge. The red line at the end of the time history is the true frequency response obtained from local numerical linearization of the nonlinear F-16 simulation. The final estimates from the real-time method fall almost exactly on the true frequency response.
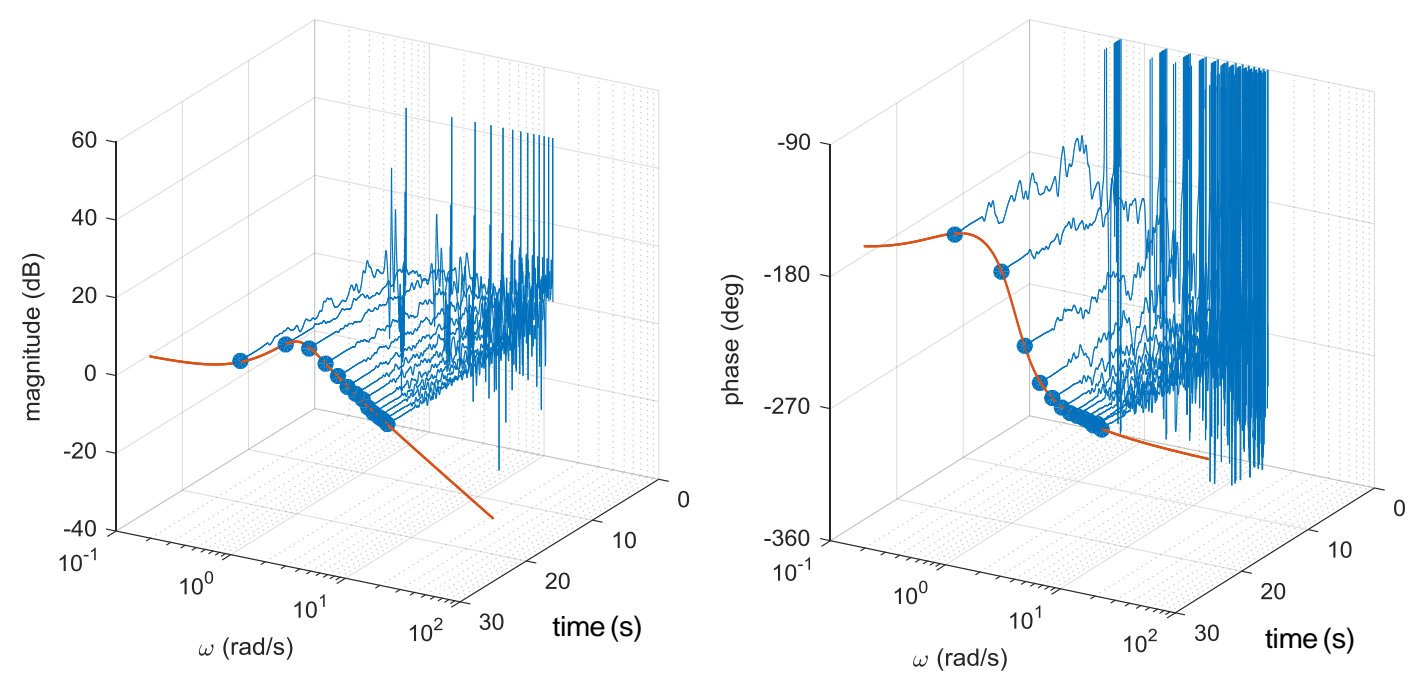

Figure 21. Real-time frequency response estimates of $q / \delta_{s}$ for the F-16 nonlinear simulation

These methods have many benefits over traditional approaches. The computations are relatively simple and few, so that many inputs, outputs, and frequencies can be processed quickly in real time using limited resources. Furthermore, because spectral density estimation techniques are not used, very few analyst judgments are needed. These techniques can therefore be used as a preliminary check in exploratory data analysis before the analysis progresses to parametric modeling. One drawback to using multisines is that only discrete frequencies are used, and any content between these frequencies is missed. However with appropriate design of the multisine inputs, the frequency spacing can be made arbitrarily small, within practical limits.

\section{Time-Domain Method}

In the first method ${ }^{27}$, harmonic functions with the excitation frequencies are fit to input and output data in the time domain. Instead of using a sinusoid with unknown amplitude and phase, a sine and cosine function are used. For example, the output in Eq. (40) is rewritten as

$$
y_{i}(t)=\sum_{k \in K} c_{k} \cos \left(\omega_{k} t\right)+d_{k} \sin \left(\omega_{k} t\right)
$$


The parameters $c_{k}$ and $d_{k}$ now appear linearly in this equation and can be estimated in real time using recursive least squares in the time domain. From these estimates, the amplitude and phase are computed as

$$
\hat{b}_{k}=\sqrt{\hat{c}_{k}^{2}+\hat{d}_{k}^{2}} \quad \hat{\psi}_{k}=\tan ^{-1}\left(d_{k} / c_{k}\right)
$$

Repeating this process for the input, estimates of the input and output amplitudes and phases are determined, and the frequency response can be computed using Eq. (41).

\section{Frequency-Domain Method}

In the second method ${ }^{28}$, sinusoids are again fit to the input and output data, but this time in the frequency domain. The Fourier transform of a sinusoid over a finite amount of time is given by Eq. (13). This equation can be rearranged, again using the output as an example, as

$$
y_{i}(\omega)=\sum_{k \in K}\left(v_{k}+\xi_{k}\right) c_{k}+j\left(v_{k}-\xi_{k}\right) d_{k}
$$

where

$$
\begin{gathered}
v_{k}=\frac{1}{2}\left(\frac{1}{\omega-\omega_{k}}\right)\left(e^{-j\left(\omega-\omega_{k}\right) t}-1\right) \\
\xi_{k}=\frac{1}{2}\left(\frac{1}{\omega+\omega_{k}}\right)\left(1-e^{-j\left(\omega+\omega_{k}\right) t}\right) \\
c_{k}=b_{k} e^{j \psi_{k}} \\
d_{k}=b_{k} e^{-j \psi_{k}} \\
\lim _{\omega \rightarrow \omega_{k}} v_{k}=-j t / 2
\end{gathered}
$$

The parameters $c_{k}$ and $d_{k}$ are estimated using sequential least squares in the frequency domain. Then amplitudes and phases can be computed using Eq. (43). An example fit to the F-16 simulation pitch rate data in the frequency domain using this method is shown in Fig. 22. Repeating this process for the input, the frequency response can be computed using Eq. (41).

This method can be viewed as the frequency-domain counterpart to the first method. The main advantage of doing the estimation in the frequency domain is that the analysis covers only the excitation bandwidth, so that highfrequency noise and other disturbances are automatically removed from the frequency-domain data. In addition, this reduces the number of data points used and therefore decreases computational requirements. There is no need for additional computation to correct the uncertainty estimates for colored residuals, as with the time-domain method. 


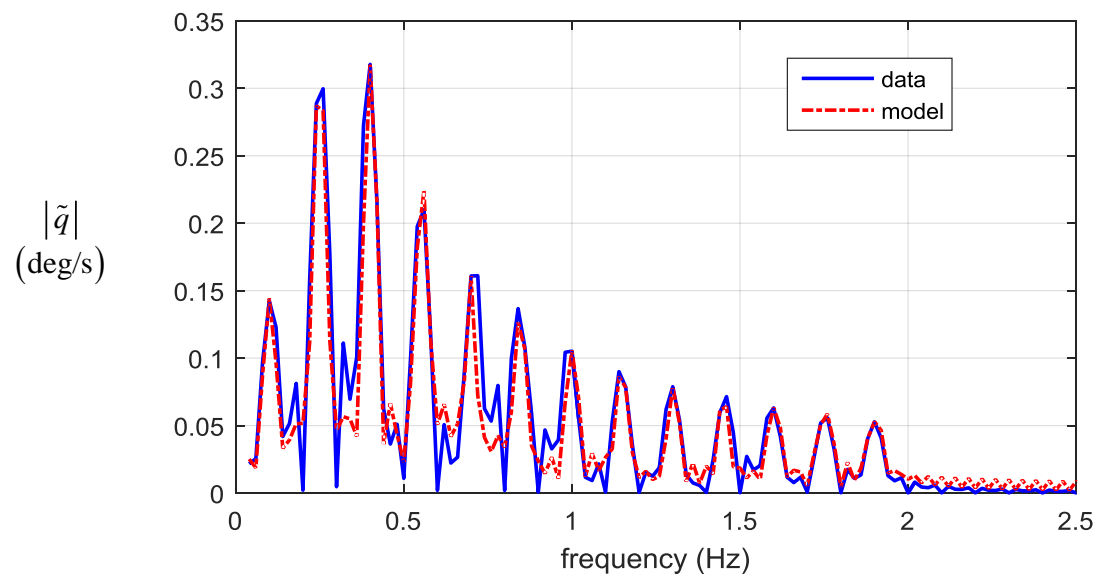

Figure 22. Model fit to pitch rate frequency-domain data for the F-16 nonlinear simulation

\section{Recursive Fourier Transform Method}

In the third method ${ }^{28,30,32}$, recursive Fourier transforms of the outputs are divided by the Fourier transforms of the inputs, as in Eq. (36), but only at the excitation frequencies $\omega_{k}$ in the multisine input. Computing frequency responses in this way is sometimes called empirical frequency response estimation, which produces poor results when the input power spectra are unknown and the data generally do not contain full periods of the input frequencies, which leads to oscillations in the estimates.

However, when multisine inputs are used, the input has power at known discrete frequencies, and the data contain full periods of the input. Under these conditions, this method gives an excellent estimate of the frequency response, as was demonstrated earlier. Only the data at the excitation frequencies $\omega_{k}$ are used to compute the frequency response, which are the peaks of the Fourier transforms and are the most important points with the highest signal-to-noise ratios. This is the same technique described earlier in Section IV.B.3, except that the recursive Fourier transform is used to produce frequency response estimates in real time.

The analysis is conducted in the frequency domain over the bandwidth of excitation, so this method has good noise and disturbance rejection. Because the computations require only arithmetic and use the fewest number of data points of the three methods, this method is the fastest and requires the least amount of computation.

References [1],[6],[15],[17]-[19] contain additional information on frequency responses, including how they can be computed accurately from measured data, and how they can be used for aircraft system identification. The next two sections provide descriptions of alternative methods using the finite Fourier transform directly that produce accurate modeling results with shorter data record requirements. These methods can be applied to nonlinear and multi-input dynamic systems such as aircraft, and are easier to use in practice.

\section{Equation-Error Modeling in the Frequency Domain}

This section shows how finite Fourier transform data can be used directly in equation-error modeling. The next section shows how finite Fourier transform data can be used in output-error modeling. Using the Fourier transform data directly, instead of first estimating spectral densities and frequency responses, avoids many of the problems discussed in Section IV, while retaining important advantages of working in the frequency domain.

\section{A. Equation-Error Parameter Estimation in the Time Domain}

Equation-error parameter estimation can be applied in the frequency domain using a similar approach to equation-error parameter estimation in the time domain. However, the simple first step of transforming the data into the frequency domain using a limited frequency band with high resolution in the frequency domain provides important practical and computational advantages.

In its most common form, the equation-error method calculates aerodynamic parameter estimates that minimize the sum of squared differences between values of nondimensional force and moment coefficients determined from measured flight data and corresponding model values. The nondimensional force and moment coefficients are computed by substituting measured and known quantities on the right sides of the following equations ${ }^{1}$ 


$$
\begin{gathered}
C_{X}=\frac{\left(m a_{x}-T\right)}{\bar{q} S} \\
C_{Y}=\frac{m a_{y}}{\bar{q} S} \\
C_{Z}=\frac{m a_{z}}{\bar{q} S} \\
C_{l}=\frac{I_{x}}{\bar{q} S b}\left[\dot{p}-\frac{I_{x z}}{I_{x}}(p q+\dot{r})+\frac{\left(I_{z}-I_{y}\right)}{I_{x}} q r\right] \\
C_{m}=\frac{I_{y}}{\bar{q} S \bar{c}}\left[\dot{q}+\frac{\left(I_{x}-I_{z}\right)}{I_{y}} p r+\frac{I_{x z}}{I_{y}}\left(p^{2}-r^{2}\right)\right] \\
C_{n}=\frac{I_{z}}{\bar{q} S b}\left[\dot{r}-\frac{I_{x z}}{I_{z}}(\dot{p}-q r)+\frac{\left(I_{y}-I_{x}\right)}{I_{z}} p q\right]
\end{gathered}
$$

This produces $N$ values of the nondimensional force and moment coefficients, where $N$ is the number of data points in the time domain. These values are often called measured force and moment coefficients, even though they not measured directly, but rather computed from other measurements and known quantities.

Model values are computed from a postulated model for each force or moment coefficient in terms of measured aircraft states and controls. Although the form of the models can vary, typical linear model structures are:

$$
\begin{gathered}
C_{X}=C_{X_{\alpha}} \alpha+C_{X_{\delta_{s}}} \delta_{s}+C_{X_{o}} \\
C_{Y}=C_{Y_{\beta}} \beta+C_{Y_{r}} \frac{r b}{2 V_{o}}+C_{Y_{\delta_{r}}} \delta_{r}+C_{Y_{o}} \\
C_{Z}=C_{Z_{\alpha}} \alpha+C_{Z_{q}} \frac{q \bar{c}}{2 V_{o}}+C_{Z_{\delta_{s}}} \delta_{s}+C_{Z_{o}} \\
C_{l}=C_{l_{\beta}} \beta+C_{l_{p}} \frac{p b}{2 V_{o}}+C_{l_{r}} \frac{r b}{2 V_{o}}+C_{l_{\delta_{a}}} \delta_{a}+C_{l_{\delta_{r}}} \delta_{r}+C_{l_{o}} \\
C_{m}=C_{m_{\alpha}} \alpha+C_{m_{q}} \frac{q \bar{c}}{2 V_{o}}+C_{m_{\delta_{s}}} \delta_{s}+C_{m_{o}} \\
C_{n}=C_{n_{\beta}} \beta+C_{n_{p}} \frac{p b}{2 V_{o}}+C_{n_{r}} \frac{r b}{2 V_{o}}+C_{n_{\delta_{a}}} \delta_{a}+C_{n_{\delta_{r}}} \delta_{r}+C_{n_{o}}
\end{gathered}
$$

For each of the equations (46a)-(47c), substituting force or moment coefficient values computed from Eqs. (48a)-(49c) on the left side, along with corresponding measured states and controls on the right, results in an over-determined set of equations for the unknown aerodynamic parameters. This problem can be solved using a standard least-squares method. 
For example, the least-squares problem for the pitching moment coefficient $C_{m}$ is formulated using the model structure in Eq. (49b) as

$$
z=\boldsymbol{X} \boldsymbol{\theta}+\boldsymbol{\varepsilon}
$$

where

$$
\begin{gathered}
\boldsymbol{z}=\left[\begin{array}{cccc}
C_{m}(1) & C_{m}(2) & \ldots & C_{m}(N)
\end{array}\right]^{T}=N \times 1 \text { vector of values computed from Eq. (47b) } \\
\boldsymbol{\theta}=\left[\begin{array}{llll}
C_{m_{\alpha}} & C_{m_{q}} & C_{m_{\delta_{s}}} & C_{m_{o}}
\end{array}\right]^{T}=4 \times 1 \text { vector of unknown parameters } \\
\boldsymbol{X}=\left[\begin{array}{llll}
\boldsymbol{\alpha} & \frac{\boldsymbol{q} \bar{c}}{2 V_{o}} & \boldsymbol{\delta}_{s} & \mathbf{1}
\end{array}\right]=N \times 4 \text { matrix of modeling function vectors or regressors } \\
\boldsymbol{\varepsilon}=\left[\begin{array}{lllll}
\varepsilon(1) & \varepsilon(2) & \ldots & \varepsilon(N)
\end{array}\right]^{T}=N \times 1 \text { vector of equation errors }
\end{gathered}
$$

The matrix $\boldsymbol{X}$ is assembled using measured data, with each column representing a modeling function, also called a regressor. The best estimator of $\boldsymbol{\theta}$ in a least-squares sense comes from minimizing the sum of squared differences between the dependent variable measurements $z$ and the model,

$$
J(\theta)=\frac{1}{2}(z-X \boldsymbol{\theta})^{T}(z-X \boldsymbol{\theta})
$$

The least-squares solution for the unknown parameter vector $\boldsymbol{\theta}$ is ${ }^{1}$

$$
\hat{\boldsymbol{\theta}}=\left(\boldsymbol{X}^{T} \boldsymbol{X}\right)^{-1} \boldsymbol{X}^{T} \boldsymbol{z}
$$

and the model output is

$$
\hat{\boldsymbol{y}}=\boldsymbol{X} \hat{\boldsymbol{\theta}}
$$

The estimated parameter covariance matrix is computed from ${ }^{1}$

$$
\begin{gathered}
\operatorname{Cov}(\hat{\boldsymbol{\theta}}) \equiv E\left[(\hat{\boldsymbol{\theta}}-\boldsymbol{\theta})(\hat{\boldsymbol{\theta}}-\boldsymbol{\theta})^{T}\right]=\hat{\sigma}^{2}\left(\boldsymbol{X}^{T} \boldsymbol{X}\right)^{-1} \equiv\left[C_{i j}\right] \quad i, j=1,2, \ldots, n_{p} \\
\hat{\sigma}^{2}=\frac{(z-\hat{\boldsymbol{y}})^{T}(z-\hat{\boldsymbol{y}})}{\left(N-n_{p}\right)}
\end{gathered}
$$

where the number of unknown parameters $n_{p}=4$ for this example. The standard errors of the estimated parameters are given by the square root of the diagonal elements of the covariance matrix,

$$
s\left(\hat{\theta}_{j}\right)=\sqrt{C_{j j}} \quad j=1,2, \ldots, n_{p}
$$

There are two important unstated assumptions underlying the expressions given above. One is that the regressors, or columns of $\boldsymbol{X}$, are assumed to be known without error. The second is that the noise on the dependent 
variable $z$ is assumed to be white and Gaussian. For practical aircraft problems, neither of these assumptions holds. However, methods have been developed to correct the problems in the time domain ${ }^{1,38}$. In contrast, if the problem is done in the frequency domain using finite Fourier transform data over a limited frequency band, then both of these practical problems disappear, as discussed next.

\section{B. Equation-Error Parameter Estimation in the Frequency Domain}

For the equation-error method applied in the time domain, when the regressors are noisy (because they are assembled from measured data) the parameter estimates will be biased ${ }^{1,38}$. However, if the problem is done in the frequency domain, the first step is applying the finite Fourier transform over a limited frequency range corresponding to the dynamics of interest. This automatically smooths the regressors, and the resulting equationerror parameter estimates are unbiased ${ }^{1,38}$. The simple first step of transforming the data into the frequency domain with a limited frequency band achieves the regressor smoothing required for unbiased parameter estimates.

A typical frequency band for the analysis of a 20 -second data record for a full-scale aircraft might be

$$
f=\left[\begin{array}{llllll}
0.100 & 0.125 & \ldots & 1.475 & 1.500
\end{array}\right]^{T} \mathrm{~Hz} \quad \omega=2 \pi f
$$

The lower bound is chosen as $2 / T$, where $T$ is the time length of the maneuver, so that the lowest analysis frequency will have 2 complete cycles in $T$ seconds, and the upper bound is chosen to include the dynamics of interest, typically identified as the upper bound of the frequency range for large components relative to the noise level in the frequency domain. Note that this upper limit can be readily found by transforming the data over a wide frequency band first (easily done with the SIDPAC fint.m program) to determine the frequency band where the large components lie.

In the frequency domain, the set of over-determined equations resulting from equation-error modeling is of the same form given in Eq. (50) above, except that the number of data points is $M$, where $M$ is the number of selected frequencies for the Fourier transformation, and the problem now involves complex numbers. The analogs of Eqs. (52)-(54) for equation-error in the frequency domain are ${ }^{1}$

$$
\begin{gathered}
\hat{\boldsymbol{\theta}}=\left[\operatorname{Re}\left(\tilde{\boldsymbol{X}}^{\dagger} \tilde{\boldsymbol{X}}\right)\right]^{-1} \operatorname{Re}\left(\tilde{\boldsymbol{X}}^{\dagger} \tilde{\boldsymbol{z}}\right) \\
\hat{\tilde{\boldsymbol{y}}}=\tilde{\boldsymbol{X}} \hat{\boldsymbol{\theta}} \\
\operatorname{Cov}(\hat{\boldsymbol{\theta}})=\tilde{G}_{\varepsilon \varepsilon}\left[\operatorname{Re}\left(\tilde{\boldsymbol{X}}^{\dagger} \tilde{\boldsymbol{X}}\right)\right]^{-1} \equiv\left[C_{i j}\right] \quad i, j=1,2, \ldots, n_{p}
\end{gathered}
$$

where $\hat{G}_{\varepsilon \varepsilon}$ is a power spectral density estimate for the residuals $\boldsymbol{\varepsilon}=\boldsymbol{z}-\boldsymbol{y}$, computed from (cf. Eqs. (27a))

$$
\hat{G}_{\varepsilon \varepsilon}(\omega)=\frac{2}{T} \tilde{\varepsilon}^{*}(\omega, T) \tilde{\varepsilon}(\omega, T) \frac{[\max (f)-\min (f)]}{f_{N}} \quad f_{N}=\frac{1}{2 \Delta t}
$$

The ratio of frequency ranges multiplying the spectral density estimate expression is needed when the analysis frequencies are concentrated in a limited frequency band within $\left[0, f_{N}\right] \mathrm{Hz}$, which can be achieved using the highaccuracy finite Fourier transform implemented in SIDPAC program fint.m. As discussed earlier, frequency resolution can be selected as $\Delta f=1 / T$ or finer, but a frequency resolution more coarse than $1 / T$ will omit frequency content and violate Parseval's theorem, Eq. (15). Data information limits dictate that the number of data points in the frequency domain cannot exceed the number of data points in the time domain, so $M \leq N$.

The standard errors of the estimated parameters are given by the square root of the diagonal elements of the covariance matrix,

$$
s\left(\hat{\theta}_{j}\right)=\sqrt{C_{j j}} \quad j=1,2, \ldots, n_{p}
$$


For the dependent variable, transforming into the frequency domain using a limited frequency band improves the signal-to-noise ratio by removing most of the wideband noise, but also leads to model residuals in the frequency domain that can be approximated as constant amplitude with frequency, which is characteristic of white noise. As a consequence, if equation-error is applied in the frequency domain with the transform frequencies chosen to approximately correspond to the large magnitude components in the frequency domain, then the calculations in Eqs. (56)-(58) can be used to accurately quantify the uncertainty in the parameter estimates.

Figure 23 shows the results from repeated application of equation-error in the frequency domain to the pitching moment equation, using simulated flight data at 5 degrees angle of attack for the F-16 aircraft with a 20-second optimized multisine applied to the stabilator. The analysis frequencies were chosen in the limited frequency band $[0.1,2.1] \mathrm{Hz}$ with frequency resolution $\Delta f=0.01 \mathrm{~Hz}$. The same maneuver was run 200 times, but with different noise sequences on the aircraft responses for every run, to simulate an ensemble of flight data. Parameter estimates for the linear model structure in Eq. (49b) are shown as $x$ markers, along with the $\pm 1 \sigma$ scatter computed from the estimates shown by the dotted lines, and the mean value of the calculated $\pm 1 \sigma$ uncertainties from Eqs. (58), shown by the dashed lines. The plots show that the calculated uncertainties characterized the scatter in the parameter estimates from repeated maneuvers very accurately.
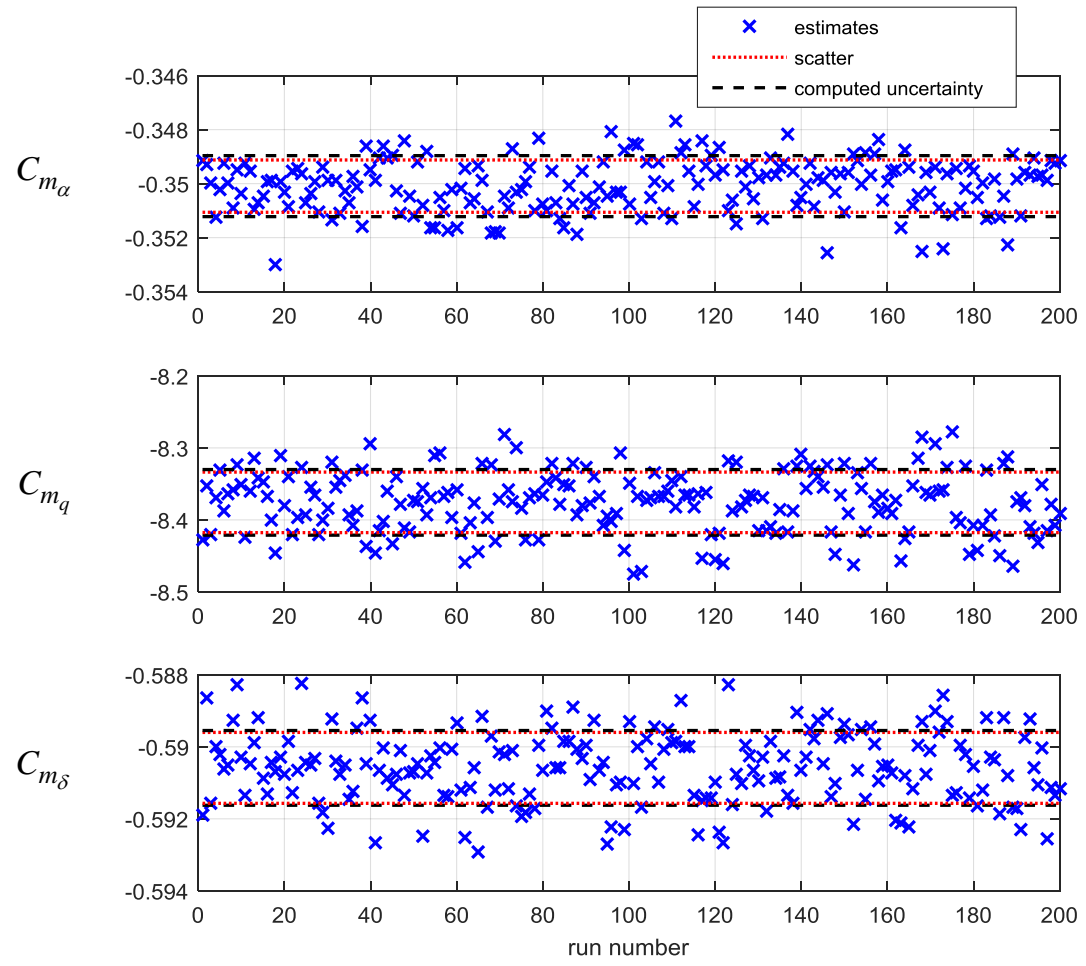

Figure 23. Model accuracy using equation-error parameter estimation calculations in the frequency domain

Note that Eqs. (56)-(57) are the same as Eqs. (52)-(53) when the data are real. Consequently, Eqs. (56)-(57) can be used for either real or complex data. When the data are complex, the expressions in Eqs. (56)-(57) are equivalent to using Eqs. (52)-(53) with the real and imaginary parts of the complex data arranged so that the imaginary values are stacked below the real parts, and treating the resulting problem as if the data were real. For example, if $\tilde{\boldsymbol{X}}=\boldsymbol{X}_{R}+j \boldsymbol{X}_{I}$, where $\boldsymbol{X}_{R}$ and $\boldsymbol{X}_{I}$ are $N \times n_{p}$ matrices of real numbers, then

$$
\operatorname{Re}\left(\tilde{\boldsymbol{X}}^{\dagger} \tilde{\boldsymbol{X}}\right)=\operatorname{Re}\left[\left(\boldsymbol{X}_{R}-j \boldsymbol{X}_{I}\right)^{T}\left(\boldsymbol{X}_{R}+j \boldsymbol{X}_{I}\right)\right]=\boldsymbol{X}_{R}^{T} \boldsymbol{X}_{R}+\boldsymbol{X}_{I}^{T} \boldsymbol{X}_{I}=\left[\begin{array}{c}
\boldsymbol{X}_{R} \\
\boldsymbol{X}_{I}
\end{array}\right]^{T}\left[\begin{array}{c}
\boldsymbol{X}_{R} \\
\boldsymbol{X}_{I}
\end{array}\right]
$$


and similarly for $\operatorname{Re}\left(\tilde{\boldsymbol{X}}^{\dagger} \tilde{z}\right)$. It follows that the regression problem for complex data can be treated as a problem with real data by simply stacking the real and imaginary parts of the complex data. However, the calculation of the covariance matrix in the frequency domain must be done according to Eqs. (58).

Equation (59) demonstrates that equation-error parameter estimation in the frequency domain treats the real and imaginary parts of the complex data equally in the least-squares solution. When the modeling is done using frequency responses, the least-squares principle is applied to magnitudes and phase angles, which are both nonlinear functions of the real and imaginary parts of the complex data. This typically results in the use of ad hoc weightings for the magnitude and phase angle contributions to the least squares cost function, see Ref. [6].

The equation-error method for parameter estimation has a relatively simple, non-iterative solution, based on linear algebra. The modeling can be done using any of the Eqs. (48)-(49) (or analogous versions of these equations, with different model structures), analyzed using one equation at a time. Because the data are detrended prior to applying the finite Fourier transform, the bias parameters, e.g., $C_{m_{o}}$ in Eq. (49b), are excluded from the analysis. Fortunately, the bias parameters can be estimated easily and accurately in the time domain, after the frequencydomain analysis is complete, as will be shown later.

The equation-error method can also be applied to state space models to estimate dimensional stability and control derivatives ${ }^{1}$, for example,

$$
\dot{q}=M_{\alpha} \alpha+M_{q} q+M_{\delta_{s}} \delta_{s}
$$

In this case, the dependent variable is $\dot{q}$, transformed into the frequency domain as described earlier, and the regressors are the transformed measured states and control. Assuming zero endpoint conditions for simplicity (Eq. (20) can be applied in the general case), the parameter estimation problem can be formulated as

$$
j \omega \tilde{q}=M_{\alpha} \tilde{\alpha}+M_{q} \tilde{q}+M_{\delta_{s}} \tilde{\delta}_{s}
$$

which is conceptually the same linear regression problem in the frequency domain discussed earlier. This is a simple and accurate method for computing important aircraft stability and control parameters very quickly. As with any modeling using dimensional derivatives as model parameters, accuracy will be affected by on how well the assumption that the model parameters are unknown constants holds in practice, given that the dimensional derivatives include dynamic pressure and mass properties.

By extension, transfer function models can also be identified in this way. For example, a transfer function model for pitch rate to stabilator deflection can be written as

$$
\frac{\tilde{q}}{\tilde{\delta}_{s}}=\frac{c_{1} s+c_{0}}{d_{2} s^{2}+d_{1} s+1}
$$

Assuming zero endpoint conditions for simplicity (Eq. (20) can be applied in the general case), and formulating the problem for equation-error parameter estimation in the frequency domain,

$$
\tilde{q}=-d_{2}(j \omega)^{2} \tilde{q}-d_{1}(j \omega) \tilde{q}+c_{1}(j \omega) \tilde{\delta}_{s}+c_{0} \tilde{\delta}_{s}
$$

After parameter estimates are obtained using least squares in the frequency domain, generating a frequency response, if that is desired, can be done by simply substituting frequency values into the transfer function model with estimated parameter values

$$
\frac{\tilde{q}}{\tilde{\delta}_{s}}=\frac{\hat{c}_{1}(j \omega)+\hat{c}_{0}}{\hat{d}_{2}(j \omega)^{2}+\hat{d}_{1}(j \omega)+1}
$$

Using the recursive Fourier transform described earlier, equation-error parameter estimation can be applied in real time, and that approach has been demonstrated in flight for many applications, such as flight envelope expansion, simulator validation from flight testing, modeling in adverse and unusual flight conditions, and health 
and safety monitoring ${ }^{11,20,22,26,33}$. Equation-error parameter estimation in the frequency domain using a limited frequency band for the Fourier transform has the advantages of applicability to either stable or unstable systems, automatic noise and dropout rejection, accurate model parameter and uncertainty estimates, and very efficient computations, without the need for long data records, single input at a time flight testing, windowing, or averaging.

The equation-error method can be considered a method wherein the model minimizes the squared error in the equations of motion, hence the name "equation error". One important practical consequence is that the equation-error method can be applied equally well to data from open-loop stable aircraft or open-loop unstable aircraft flying under closed-loop feedback control. No integration of the equations of motion is necessary, and the computations needed for the optimization are relatively simple and non-iterative. However, the equation-error approach usually matches state time-derivative information from the dynamic system, rather than matching the states or outputs, as in the output-error method. This can be seen from the right sides of Eqs. (46)-(47), where the quantities used as dependent variables in the equation-error method are computed from translational and angular accelerations. Consequently, a model that uses equation-error parameter estimates will not produce the best match to the measured outputs of the dynamic system, since that is not what is being optimized.

This section discussed the model parameter estimation problem, where the model structure (terms to be included in the model) was specified. Model structure determination methods that have been developed and used successfully in the time domain can be also be applied in the frequency domain, as will be discussed later. However, with the efficient real-time equation-error parameter estimation capability in the frequency domain, the model structure determination problem can be bypassed by assuming a fixed linear model structure, but allowing the model parameters to be time-varying and estimating those time-varying parameters in real time. This approach has many practical applications and has been demonstrated in flight ${ }^{1,5,10,11,20,22,26,35,36}$.

\section{Bias and Trend Estimation in the Time Domain}

Because the time series for the model terms and the dependent variable are detrended prior to Fourier transformation, the bias term in the model and the trend with time are not observable using frequency-domain modeling. Stated another way, frequency-domain data include only dynamic information content, and not static or trend information. Consequently, a separate and subsequent step must be included to update the model bias and trend with time. The estimated bias $\hat{\boldsymbol{\theta}}_{o}$ then corresponds to the aerodynamic bias term in a multivariable Taylor series expansion for the aerodynamic coefficient, which is generally of most interest for aerodynamics and flight dynamics.

Fortunately, this step is easy to accomplish in the time domain, because the bias and trend terms are typically among the most well-conditioned of all model terms, and therefore are easy to estimate accurately in the time domain. The approach could be considered the reverse of the process described earlier for detrending the data prior to applying the finite Fourier transform.

After the frequency-domain modeling is complete, the estimated model parameter vector $\hat{\boldsymbol{\theta}}$ is used to estimate the model bias and trend parameters and associated covariance as follows (using a linear trend as an example):

$$
\begin{gathered}
\boldsymbol{\theta}_{d}=\left[\begin{array}{c}
\theta_{o} \\
\theta_{t}
\end{array}\right] \quad \boldsymbol{X}_{d}=\left[\begin{array}{ll}
\mathbf{1} & \boldsymbol{t}
\end{array}\right] \\
\hat{\boldsymbol{\theta}}_{d}=\left(\boldsymbol{X}_{d}^{T} \boldsymbol{X}_{d}\right)^{-1}\left[\boldsymbol{X}_{d}^{T}(\boldsymbol{z}-\boldsymbol{X} \hat{\boldsymbol{\theta}})\right] \\
\Sigma_{\hat{\boldsymbol{\theta}}_{d}}=\hat{\sigma}_{d}^{2}\left(\boldsymbol{X}_{d}^{T} \boldsymbol{X}_{d}\right)^{-1} \\
\hat{\sigma}_{d}^{2}=\frac{1}{(N-2)}\left[\left(z-\boldsymbol{X} \hat{\boldsymbol{\theta}}-\boldsymbol{X}_{d} \hat{\boldsymbol{\theta}}_{d}\right)^{T}\left(z-\boldsymbol{X} \hat{\boldsymbol{\theta}}-\boldsymbol{X}_{d} \hat{\boldsymbol{\theta}}_{d}\right)\right]
\end{gathered}
$$

This process can be viewed as restoring the bias and trend information that was removed prior to Fourier transformation, so that the model is correct in the time domain. The time-domain bias and trend update is done after equation-error modeling in the frequency domain is complete, holding those estimated model parameters fixed. 


\section{Modulating Function Approach}

The finite Fourier transform of time derivatives involves endpoint corrections when the time series endpoints and/or derivatives are non-zero, as shown earlier in Eq. (20). These endpoint corrections can be avoided using modulating functions, in a manner first described by Shinbrot ${ }^{39}$. The general idea is to multiply a differential equation by suitably-chosen modulating functions, then use integration by parts to transfer the derivatives from the data to the modulating functions, incurring endpoint terms that involve the modulating functions and their time derivatives evaluated at the endpoints. A smooth differentiable function $\phi(t)$ with the value of the function and its first $n-1$ time derivatives equal to zero at the endpoints is called an $n$ th-order modulating function,

$$
\phi^{(k)}(0)=\phi^{(k)}(T)=0 \quad k=0,1, \ldots, n-1
$$

where $\phi^{(1)}(t)$ denotes the first time derivative of $\phi(t)$, and so on. By transferring the endpoint terms to the modulating functions, the endpoints conditions in the data are removed from the problem, so that the endpoint conditions in the data can be arbitrary.

Pearson ${ }^{40}$ developed a Fourier-based modulating function. The Fourier-based modulating function for frequency $\omega$ is

$$
\phi(t)=e^{-j \omega t}\left(1-e^{-j \omega_{o} t}\right)^{n} \quad \omega_{o} \equiv 2 \pi / T
$$

The function $\phi(t)$ in Eq. (68) satisfies the requirements of Eq. (67) for an $n$ th-order modulating function. This modulating function can also be written as

$$
\phi(t)=\sum_{k=0}^{n} b_{k} e^{-j\left(\omega+k \omega_{o}\right) t} \quad b_{k}=(-1)^{k} \frac{n !}{k !(n-k) !}
$$

which shows that the modulating function $\phi(t)$ is a summation of complex sinusoidal functions of the same type used in the finite Fourier transform of Eq. (2).

For example, starting with the $n$ th-order transfer function

$$
\frac{\tilde{y}}{\tilde{u}}=\frac{c_{m} s^{m}+c_{m-1} s^{m-1}+\ldots+c_{1} s+c_{0}}{d_{n} s^{n}+d_{n-1} s^{n-1} \ldots+d_{1} s+1}
$$

The associated differential equation is

$$
d_{n} y^{(n)}+d_{n-1} y^{(n-1)} \ldots+d_{1} y^{(1)}+y=c_{m} u^{(m)}+c_{m-1} u^{(m-1)} \ldots+c_{1} u^{(1)}+c_{0} u+\varepsilon
$$

where $\varepsilon$ is the equation error incurred when using this model to characterize measured data. Multiplying by $\phi(t)$, and integrating over the time period $[0, T]$,

$$
\begin{aligned}
\int_{0}^{T} \phi y d t= & c_{0} \int_{0}^{T} \phi u d t+c_{1} \int_{0}^{T} \phi u^{(1)} d t+\ldots+c_{m-1} \int_{0}^{T} \phi u^{(m-1)} d t+c_{m} \int_{0}^{T} \phi u^{(m)} d t \\
& -d_{1} \int_{0}^{T} \phi y^{(1)} d t-d_{2} \int_{0}^{T} \phi y^{(2)} d t-\ldots-d_{n-1} \int_{0}^{T} \phi y^{(n-1)} d t-d_{n} \int_{0}^{T} \phi y^{(n)} d t+\tilde{\varepsilon}
\end{aligned}
$$

where $\tilde{\varepsilon}$ represents the complex-valued equation error for the modulated equation. Using integration by parts, and invoking the properties of an $n$ th-order modulating function from Eq. (67), 


$$
\begin{aligned}
\int_{0}^{T} \phi y d t= & c_{0} \int_{0}^{T} \phi u d t-c_{1} \int_{0}^{T} \phi^{(1)} u d t+\ldots+c_{m-1}(-1)^{m-1} \int_{0}^{T} \phi^{(2)} u d t+c_{m}(-1)^{m} \int_{0}^{T} \phi^{(m)} u d t \\
& +d_{1} \int_{0}^{T} \phi^{(1)} y d t-d_{2} \int_{0}^{T} \phi^{(2)} y d t+\ldots-d_{n-1}(-1)^{n-1} \int_{0}^{T} \phi^{(n-1)} y d t-d_{n}(-1)^{n} \int_{0}^{T} \phi^{(n)} y d t+\tilde{\varepsilon}
\end{aligned}
$$

Each term in Eq. (72) is a complex-valued modulation of the input-output data for frequency $\omega$. The $i$ th time derivative of the modulating function $\phi(t)$ is

$$
\phi^{(i)}(t)=\sum_{k=0}^{n} b_{k}\left[-j\left(\omega+k \omega_{o}\right)\right]^{i} e^{-j\left(\omega+k \omega_{o}\right) t} \quad b_{k}=(-1)^{k} \frac{n !}{k !(n-k) !}
$$

Using Eq. (2), the modulation of the output data $y$ can be carried out using the high-accuracy finite Fourier transform with arbitrary frequencies implemented in SIDPAC program fint.m as

$$
\begin{aligned}
\int_{0}^{T} \phi^{(i)} y d t & =\sum_{k=0}^{n} b_{k}\left[-j\left(\omega+k \omega_{o}\right)\right]^{i} \int_{0}^{T} y e^{-j\left(\omega+k \omega_{o}\right) t} d t \\
& =\sum_{k=0}^{n} b_{k}\left[-j\left(\omega+k \omega_{o}\right)\right]^{i} \tilde{y}\left(\omega+k \omega_{o}\right)
\end{aligned}
$$

and similarly for the input data $u$.

Carrying out this process for a vector of frequencies $\omega$ provides frequency-domain data of the same type obtained earlier using the finite Fourier transform. However, using the finite Fourier transform required detrending the data or assuming that the endpoint conditions were close to zero. Using the modulating function approach, that assumption is not required, because the modulation process removes the endpoint terms analytically. The modulating function approach therefore allows the use of input-output time series data with arbitrary endpoint conditions and no detrending is required.

Figure 24 shows simulated F-16 data for stabilator input and pitch rate output. A known transfer function model was used to generate the data, so that the true model parameters were known. Magnitude and slope discontinuities at the endpoints were introduced deliberately by adding an arbitrary offset and linear trend with time to the input. The modulating function approach was applied to this data, using the frequency vector

$$
f=\left[\begin{array}{lllll}
0.1 & 0.2 & \ldots & 1.7 & 1.8
\end{array}\right]^{T} \mathrm{~Hz} \quad \omega=2 \pi f
$$

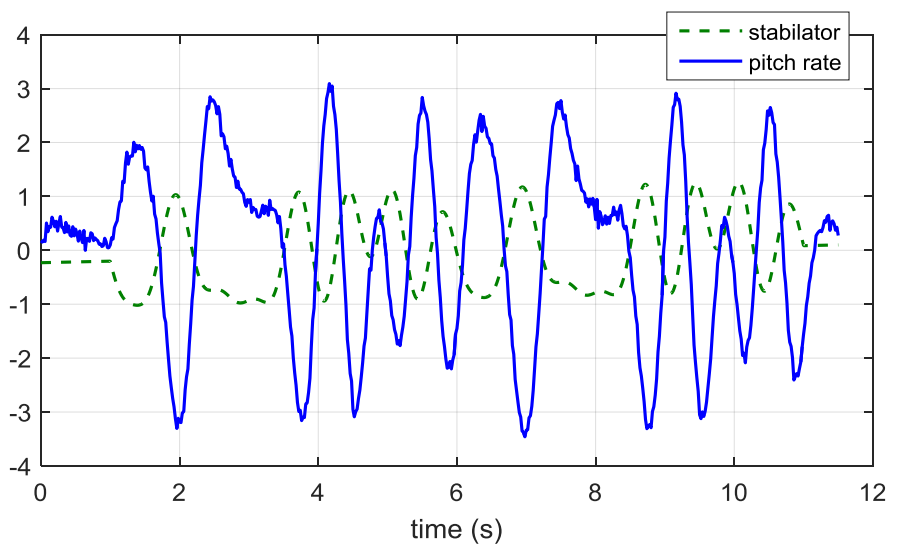

Figure 24. Simulated F-16 input-output data 
Figures 25 and 26 show model fits to the data in the time and frequency domains, respectively. The parameter estimation results in Table 2 show that the model parameter estimates are correct within random statistical error.
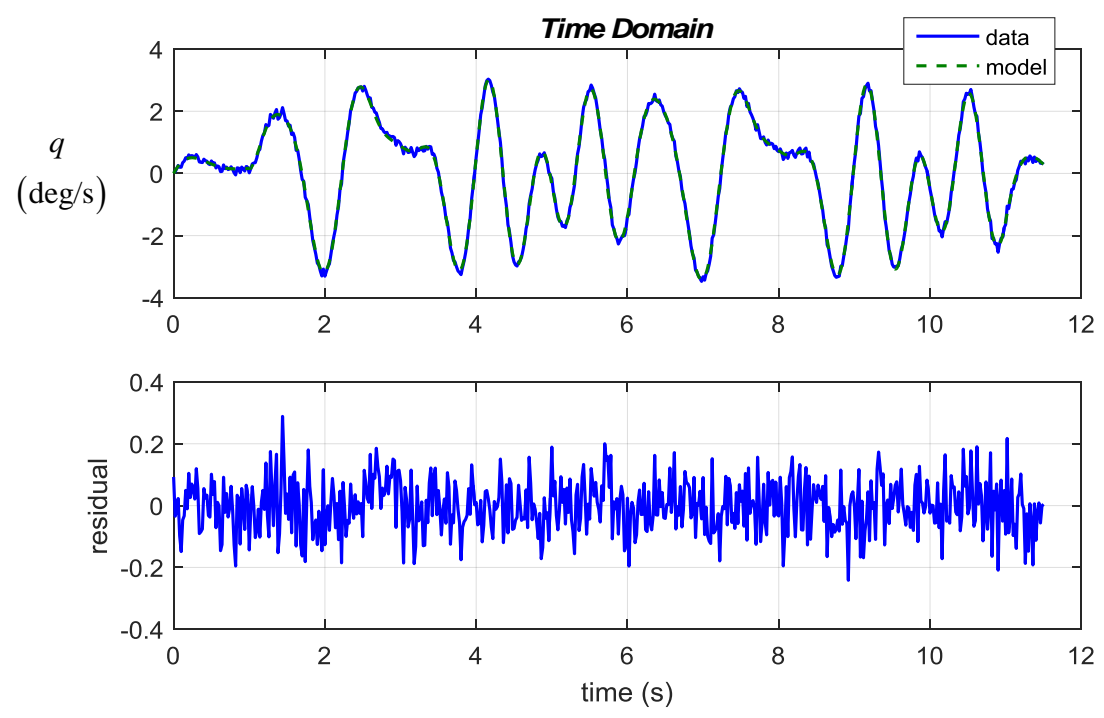

Figure 25. Modulating function model fit in the time domain
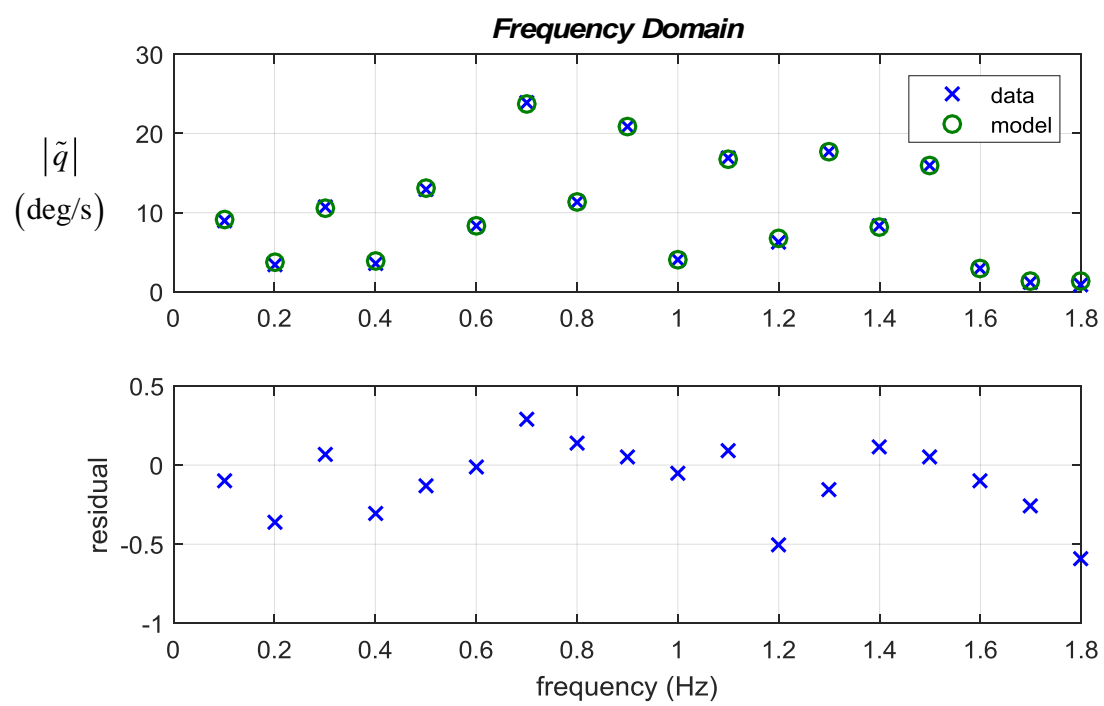

Figure 26. Modulating function model fit in the frequency domain

The modulating function approach is similar to applying equation-error in the frequency domain, except that using modulating functions does not require detrending the time-domain data and is robust to non-zero endpoint conditions.

\section{E. Nonlinear Model Terms}

Nonlinear model terms can be used for equation-error modeling in the frequency domain, if the proper data conditioning is applied in the time domain prior to transformation to the frequency domain. This works because the finite Fourier transform is implemented numerically, and therefore it makes no difference how the time series was created. The least-squares solution used for equation-error modeling depends on the linearity of the model output with respect to the parameters only, so the model terms can be nonlinear. There are important practical advantages associated with nonlinear model terms in the frequency domain, as will be shown. 
When assembling polynomial nonlinear model terms in the time domain, an important issue arises concerning model term correlation. This can be understood from a simple example, where a quadratic term in angle of attack is to be included as a model term. The angle of attack time series from a flight test maneuver can be considered to be composed of two parts - a steady part $\alpha_{o}$ and a dynamic part $\Delta \alpha$,

$$
\alpha=\alpha_{o}+\Delta \alpha
$$

The quadratic term in angle of attack is then

$$
\alpha^{2}=\left(\alpha_{o}+\Delta \alpha\right)\left(\alpha_{o}+\Delta \alpha\right)=\alpha_{o}^{2}+2 \alpha_{o} \Delta \alpha+\Delta \alpha^{2}
$$

If the steady part of the angle of attack is not removed, a quadratic term includes contributions that are relatively constant $\left(\alpha_{o}^{2}\right)$, and also linear in the dynamic part $\left(2 \alpha_{o} \Delta \alpha\right)$, along with the intended quadratic dynamic part $(\Delta \alpha)^{2}$. This confounds the quadratic term with both the constant and the linear term in angle of attack, and these terms may already be included in the model. In the time domain, the result is correlation among the model terms, and compromised model accuracy. However, if the angle of attack time series is detrended first, as is done prior to applying the finite Fourier transform, then the relatively constant part is removed, and the problem is avoided. The square of a detrended angle of attack time series simply results in a different time series, which can be transformed to the frequency domain like any other time series.

The same issue occurs regardless of the number of explanatory variables involved or the order of the polynomial nonlinearity. In general, any polynomial term will be confounded with lower-order polynomial terms unless the steady parts are removed from all explanatory variable data first.

However, polynomial terms assembled from detrended explanatory variable data can still have biases and linear trends with time, because of the nonlinearities involved in the multiplications. Therefore, the procedure is to detrend the explanatory variable data first, then assemble the nonlinear terms in the time domain, then detrend the resulting time series data and apply the finite Fourier transform as usual. This solves the modeling function correlation issue in the time domain prior to applying the Fourier transform, and makes it possible to include polynomial nonlinear terms in the frequency domain.

Another advantage of modeling in the frequency domain using nonlinear model terms is the ability to identify frequency-dependent aerodynamic effects. This has been investigated in connection with identifying aerodynamic transfer functions ${ }^{41}$, which are a generalization of traditional stability and control derivatives. A simple example can be used to demonstrate the capability.

The dependence of unsteady aerodynamic lift on angle of attack can be characterized using an indicial function model as follows ${ }^{42,43}$

$$
\begin{gathered}
C_{L_{u}}=-a x=-a\left[\int_{0}^{t} e^{-b(t-\tau)} \dot{\alpha}(\tau) d \tau\right] \\
x \equiv \int_{0}^{t} e^{-b(t-\tau)} \dot{\alpha}(\tau) d \tau
\end{gathered}
$$

where $C_{L_{u}}$ denotes the unsteady part of the lift coefficient, and $x$ is an additional state associated with the unsteady aerodynamics. Model parameters $a$ and $b$ in general depend on the nominal angle of attack $\alpha_{o}$, and the indicial function is the simple exponential function $e^{-b t}$. The expression for $C_{L_{u}}$ describes an addition to the lift coefficient that depends on past values of $\dot{\alpha}$, but fades away with time. The unsteady aerodynamics $C_{L_{u}}$ is added to the quasisteady lift coefficient normally obtained from wind tunnel tests. In the above equations, the $b$ parameter quantifies the extent of the lag effect (due to past values of $\dot{\alpha}$ ), and the $a$ parameter quantifies a conventional quasi-static $\dot{\alpha}$ effect. 
Applying the finite Fourier transform to Eq. (78), using a power series expansion for the indicial function $e^{-b(t-\tau)}$, assuming approximately steady initial and final conditions so that a time derivative becomes a simple multiplication by $j \omega$ in the frequency domain, and noting that the Fourier transform of a time convolution of two functions equals the multiplication of their Fourier transforms ${ }^{1}$,

$$
\begin{gathered}
\mathcal{F}\left[\int_{0}^{t} e^{-b(t-\tau)} \dot{\alpha}(\tau) d \tau\right]=\mathcal{F}\left\{\int_{0}^{t}\left[1-b(t-\tau)+\frac{b^{2}(t-\tau)^{2}}{2 !}-\frac{b^{3}(t-\tau)^{3}}{3 !}+\ldots\right] \dot{\alpha}(\tau) d \tau\right\} \\
\mathcal{F}\left[\int_{0}^{t} e^{-b(t-\tau)} \dot{\alpha}(\tau) d \tau\right]=\left[1-\frac{b}{j \omega}+\frac{b^{2}}{(j \omega)^{2}}-\frac{b^{3}}{(j \omega)^{3}}+\ldots\right] \tilde{\alpha}
\end{gathered}
$$

This expression shows that a simple unsteady effect can be modeled in the frequency domain by a power series in $(j \omega)^{-1}$ multiplied by the Fourier transform of the explanatory variable, which is angle of attack in this case. A reasonable approximation in the frequency domain can be obtained by truncating the power series after a few terms.

Other nonlinear model terms built from multiplying the Fourier transform of angle of attack by powers of both $j \omega$ and $(j \omega)^{-1}$ make the model structure even more general, and is equivalent to allowing for a more complicated indicial function than $e^{-b t}$.

This approach can be extended by using both positive and negative powers of $j \omega$ multiplying the Fourier transform of other explanatory variables, such as sideslip angle or nondimensional pitch rate.

\section{F. Orthogonalization and Model Structure Determination in the Frequency Domain}

Capable and practical techniques have been developed in the time domain to determine model structure (i.e., selecting which terms should be included in the model) using orthogonal modeling functions computed from the data ${ }^{1}$. In the frequency domain, the data are complex numbers. This requires slightly different data handling to achieve orthogonalization, but otherwise, the same orthogonalization procedure and modeling approaches used for time-domain data can be applied.

The necessary modification for orthogonalizing complex data vectors is simple, and is related to what is called the Euclidean angle between two complex vectors ${ }^{44}$. For an arbitrary complex vector $\boldsymbol{x}_{C}$ with $M$ generally complex elements, a real isometric vector $\boldsymbol{x}_{R}$ with $2 M$ elements can be assembled by stacking the real and imaginary parts of the complex elements of $\boldsymbol{x}_{C}$ :

$$
\boldsymbol{x}_{R}=\left[\begin{array}{c}
\operatorname{Re}\left[\boldsymbol{x}_{C}\right] \\
\operatorname{Im}\left[\boldsymbol{x}_{C}\right]
\end{array}\right]
$$

Treating the real vector $\boldsymbol{x}_{R}$ as the new data vector, all of the orthogonalization techniques and model structure determination techniques developed previously for real data in the time domain can be applied without modification. The penalty associated with applying the techniques to complex data is simply a doubling of the length of the data vectors involved. The measured response data in the frequency domain becomes a $2 \mathrm{M}$-dimensional real vector,

$$
z=\left[\begin{array}{l}
\operatorname{Re}[\tilde{z}(\omega)] \\
\operatorname{Im}[\tilde{z}(\omega)]
\end{array}\right]
$$

where $\tilde{z}(\omega)$ is the complex vector of Fourier transforms for $M$ selected frequencies in the frequency vector $\boldsymbol{\omega}$.

The candidate model terms for orthogonalization are generated by computing the (generally nonlinear) model terms in the time domain using detrended explanatory variable data, detrending the resulting time series data, applying the Fourier transform, then converting the resulting frequency-domain data to real data, 


$$
\boldsymbol{X}=\left[\begin{array}{l}
\operatorname{Re}[\tilde{\boldsymbol{X}}(\boldsymbol{\omega})] \\
\operatorname{Im}[\tilde{\boldsymbol{X}}(\boldsymbol{\omega})]
\end{array}\right]
$$

where $\tilde{\boldsymbol{X}}(\boldsymbol{\omega})$ is the complex matrix of Fourier transforms of the candidate model terms for $M$ selected frequencies in the frequency vector $\boldsymbol{\omega}$. From this point, the model structure determination for identifying model terms from flight data can use the same approaches developed and used successfully in the time domain ${ }^{1,20,21,45}$. References [41] and [46] demonstrate practical application of nonlinear regressors and model structure determination in the frequency domain to transfer function identification, unsteady aerodynamic modeling, and automated simulation updating based on flight data.

\section{Output-Error Modeling in the Frequency Domain}

Output-error parameter estimation can be performed using frequency-domain data ${ }^{1-3}$, but only if the dynamic model is linear. This follows from the linearity of the finite Fourier transform defined in Eq. (2). In the frequencydomain output-error formulation, a modified Newton-Raphson optimization technique is employed to determine the model parameters that best fit the model outputs to the measured outputs in the frequency domain. Analysis in the frequency domain can be beneficial because frequency-domain data can be restricted to only the bandwidth of interest, and unstable systems can be identified without any modification of the method. Additionally, the computations are much faster using frequency-domain data because fewer data points are used in the analysis, convolution in the time domain becomes multiplication in the frequency domain, and estimated parameter uncertainties do not need to be corrected for colored residuals ${ }^{1}$.

\section{A. Linearity Requirement}

Output-error modeling using frequency-domain data is restricted to linear, time-invariant systems. Using statespace notation, these equations are written in the canonical form as

$$
\begin{aligned}
& \dot{\boldsymbol{x}}(t)=\boldsymbol{A} \boldsymbol{x}(t)+\boldsymbol{B} \boldsymbol{u}(t) \\
& \boldsymbol{y}(t)=\boldsymbol{C} \boldsymbol{x}(t)+\boldsymbol{D} \boldsymbol{u}(t)
\end{aligned}
$$

where $\boldsymbol{x}(t)$ and $\boldsymbol{u}(t)$ are the state and input vectors, and $\boldsymbol{A}, \boldsymbol{B}, \boldsymbol{C}$, and $\boldsymbol{D}$ are the system matrices. The system matrices must have elements that are constants so that the Fourier transform can be applied to the dynamic equations. The state vector solution in the frequency domain is

$$
\tilde{\boldsymbol{x}}(\omega)=(j \omega \boldsymbol{I}-\boldsymbol{A})^{-1} \boldsymbol{B} \tilde{\boldsymbol{u}}(\omega)
$$

with outputs

$$
\begin{gathered}
\tilde{\boldsymbol{y}}(\omega)=\boldsymbol{C} \tilde{\boldsymbol{x}}(\omega)+\boldsymbol{D} \tilde{\boldsymbol{u}}(\omega) \\
\tilde{\boldsymbol{y}}(\omega)=\left[\boldsymbol{C}(j \omega \boldsymbol{I}-\boldsymbol{A})^{-1} \boldsymbol{B}+\boldsymbol{D}\right] \tilde{\boldsymbol{u}}(\omega)
\end{gathered}
$$

Note that the computation of model outputs in Eq. (87) uses only matrix multiplications. The corresponding solution in the time domain involves a convolution integral, which is significantly more complex and requires more computation, and there are typically fewer data points in the frequency domain than in the time domain, so the output-error optimization executes faster in the frequency domain.

\section{B. Bias Estimation}

As discussed earlier, the bias and trend are removed from time-domain data before applying the Fourier transform to reduce spectral leakage at low frequencies. Consequently, there are fewer parameters to estimate with 
output error when using frequency-domain data compared to time-domain data, which results in fewer iterations for convergence and fewer starting values for model parameters. However, this also means that bias and trend information cannot be estimated in the frequency domain and a second step using time-domain data is required, similar to the discussion for equation-error given earlier. Bias parameter estimates can be important modeling results in some cases, and are also required if time-domain model fits or prediction cases are used to verify the model.

Given estimates of the parameters in the system matrices, a second output-error analysis can be formulated in the time domain as

$$
\begin{aligned}
& \dot{\boldsymbol{x}}(t)=\boldsymbol{A} \boldsymbol{x}(t)+\boldsymbol{B} \boldsymbol{u}(t)+\boldsymbol{b}_{\dot{\boldsymbol{x}}} \\
& \boldsymbol{y}(t)=\boldsymbol{C} \boldsymbol{x}(t)+\boldsymbol{D} \boldsymbol{u}(t)+\boldsymbol{b}_{\boldsymbol{y}}
\end{aligned}
$$

where the unknown parameters to be estimated are

$$
\boldsymbol{\theta}=\left[\begin{array}{l}
\boldsymbol{b}_{\dot{x}} \\
\boldsymbol{b}_{\boldsymbol{y}}
\end{array}\right]
$$

The state equation biases $\boldsymbol{b}_{\dot{\boldsymbol{x}}}$ represent steady-state inputs in the state equations, for example, from non-zero reference conditions, or unmodeled steady wind. The output biases $\boldsymbol{b}_{\boldsymbol{y}}$ account for systematic instrumentation errors, such as bias errors in accelerometer measurements. Generally, the bias parameter estimation problem is wellconditioned and applying time-domain output-error estimation with parameters in the system matrices held fixed at the values estimated during the frequency-domain output-error analysis, provides accurate bias parameter estimates quickly and easily.

\section{Starting Values and Convergence}

Output-error parameter estimation requires nonlinear optimization, and therefore needs good starting values for the model parameters. Otherwise, the estimator may use an excessive number of iterations to converge, may converge to an incorrect local minimum, or may diverge. Starting values can come from different data, a separate analysis such as equation-error parameter estimation, or practical experience.

Important quantities in the optimization are the output sensitivities, which quantify the changes in the model outputs due to changes in each parameter. If a parameter has a low output sensitivity, large changes can be made to the parameter without significantly altering the model outputs, which results in poor identifiability of the parameter. Output sensitivities are typically computed numerically using finite differences, to avoid deriving the sensitivity equations for every model structure considered. However, output-error parameter estimation in the frequency domain is restricted to linear systems, which all have the output sensitivity equations ${ }^{1,2,47}$

$$
\frac{\partial \tilde{\boldsymbol{y}}(\omega)}{\partial \boldsymbol{\theta}}=\boldsymbol{C}(j \omega \boldsymbol{I}-\boldsymbol{A})^{-1}\left[\frac{\partial \boldsymbol{A}}{\partial \boldsymbol{\theta}} \tilde{\boldsymbol{x}}(\omega)+\frac{\partial \boldsymbol{B}}{\partial \boldsymbol{\theta}} \tilde{\boldsymbol{u}}(\omega)\right]+\frac{\partial \boldsymbol{C}}{\partial \boldsymbol{\theta}} \tilde{\boldsymbol{x}}(\omega)+\frac{\partial \boldsymbol{D}}{\partial \boldsymbol{\theta}} \tilde{\boldsymbol{u}}(\omega)
$$

During the optimization, the states $\tilde{\boldsymbol{x}}(\omega)$ substituted in the sensitivity equation come from solving the state equations using the current parameter estimate vector $\boldsymbol{\theta}$. However, if measurements of all the states are available, as is often the case in aircraft flight testing, the transformed measured states can be substituted into the output sensitivity equations. The resulting iteration based on output sensitivities computed in this way will typically produce parameter estimates very close to the solution. Using this procedure for the first iteration, and afterwards proceeding normally, effectively removes the need for starting values on the model parameters and reduces the number of iterations for convergence. The cost for doing this is a slight increase in code complexity to handle the

startup iteration, and the requirement that all the states are measured. This method was first presented by Denery ${ }^{48}$, was implemented in early output-error software ${ }^{49}$, and has been employed for real-time data compatibility analysis ${ }^{47}$. 


\section{Prior Information and Data from Multiple Maneuvers}

Prior information on model parameters can be specified in the output-error method ${ }^{1}$. This improves the conditioning of the information matrix, and weights the prior information relative to the information in the data used during the estimation, essentially based on statistical analysis and multiple output signal-to-noise ratios.

This situation can arise if information is available on a parameter to mitigate identifiability problems. For example, in normal flight test maneuvers at low nominal angle of attack, pitch rate and angle of attack rate are highly correlated, so that any associated model parameters are not separately identifiable. If a mean value and variance for a model parameter associated with one of these quantities can be determined from a separate analysis or prior information, the remaining parameter can be estimated from the flight test data. Another application is realtime estimation using output-error ${ }^{47}$, where the time series data are segmented into smaller sections, and the estimator is applied sequentially, using prior information for the model parameters and covariances from the previous segment.

Fourier transform data from multiple maneuvers can be combined to estimate one set of model parameters. This is useful if the data sets contain good information content on different parameters, or if one set of model parameters is desired from several maneuvers. Fourier transform data from multiple maneuvers can be simply added for a combined output-error analysis, assuming that the model structure, noise covariances, and analysis frequencies are consistent throughout all maneuvers. If the model parameter estimates should be different between maneuvers (e.g., a maneuver at low and high nominal angles of attack), an averaged estimate based on the data information content will result. Likewise, an averaged result will be obtained if the noise covariances are significantly different between maneuvers. Consistent analysis frequencies can be implemented using the chirp $z$-transform, as discussed earlier, even for maneuvers with different time durations.

\section{Concluding Remarks}

Several analytical and practical issues related to applying frequency-domain methods to aircraft dynamic modeling problems were examined and discussed. Methods and techniques studied included transforming timedomain data into the frequency domain, detrending, data analysis in the frequency domain, real-time Fourier transformation, frequency response estimation, equation-error and output-error parametric modeling in the frequency domain, and real-time methods. Advantages, limitations, and deficiencies of frequency-domain methods were discussed.

Practical issues were examined using data from an F-16 nonlinear simulation, with realistic noise sequences added to the computed aircraft responses. This allowed a clear view of the effectiveness of various frequencydomain approaches to data analysis and modeling problems, because the true system characteristics were known. This strategy is necessary to fully understand and validate tools for flight data analysis and modeling.

Some issues were illustrated using flight data from the T-2 subscale generic transport aircraft. Although the issues and methods were presented and demonstrated for fixed-wing aircraft, the same methods can be applied to other aircraft types as well, such as rotorcraft, unmanned air vehicles (UAV), space vehicles, and dynamic systems in general.

Software tools for all of the techniques and investigations, along with many other methods used in aircraft system identification, can be found in the MATLAB $^{\circledR}$ software package called SIDPAC (System IDentification Programs for $\underline{\text { AirCraft }})^{1,12,13}$.

Frequency-domain methods have many distinct and important advantages compared to time-domain methods, including reduced dimensionality from the projection of the data onto sinusoidal basis functions, improved noise rejection, simplified methods for accurate modeling and model uncertainty characterization, more effective approaches to data filtering and smoothing, and useful viewpoints for both the data and the modeling results. Mainly because the data are transformed, there are many pitfalls that await the unwary when applying frequency-domain methods, so that a good understanding of the fundamental issues is required to use frequency-domain methods effectively. Recently-developed, useful frequency-domain techniques were explained and demonstrated, with some discussion and comparisons with conventional frequency-domain methods. Frequency-domain methods, implemented using modern analytical and numerical techniques, and applied with knowledge of their workings, are extremely useful tools for solving aircraft dynamic modeling problems.

\section{Acknowledgments}

The efforts of the AirSTAR flight test team at NASA Langley in building and testing the T-2 aircraft and associated systems, carefully calibrating the instrumentation, and carrying out the flight operations to collect the high-quality flight data used in this study, are gratefully acknowledged. Research in Aircraft System Identification is 
funded by the NASA Aviation Safety Program, Vehicle Systems Safety Technologies (VSST) project and the NASA Transformational Tools and Technologies (TTT) project.

\section{References}

${ }^{1}$ Morelli, E.A. and Klein, V. Aircraft System Identification - Theory and Practice, $2^{\text {nd }}$ Edition, Sunflyte Enterprises, Williamsburg, VA, December 2016.

${ }^{2}$ Klein, V. "Aircraft Parameter Estimation in Frequency Domain," AIAA Paper 78-1344, AIAA Atmospheric Flight Mechanics Conference, Palo Alto, CA, August 1978.

${ }^{3}$ Klein, V. "Maximum Likelihood Method for Estimating Airplane Stability and Control Parameters From Flight Data in Frequency Domain,” NASA TP-1637, May 1980.

${ }^{4}$ Morelli, E.A. "Identification of Low Order Equivalent System Models from Flight Test Data," NASA TM-2000-210117, August 2000.

${ }^{5}$ Morelli, E.A. "Real-Time Parameter Estimation in the Frequency Domain," Journal of Guidance, Control, and Dynamics, Vol. 23, No. 5, September-October 2000, pp. 812-818.

${ }^{6}$ Tischler, M.B. and Remple, R.K. Aircraft and Rotorcraft System Identification - Engineering Methods with Flight Test Examples, $2^{\text {nd }}$ Edition, AIAA Education Series, AIAA, Reston, VA, 2012.

${ }^{7}$ Garza, F.R. and Morelli, E.A. "A Collection of Nonlinear Aircraft Simulations in MATLAB," NASA / TM-2003-212145, January 2003.

${ }^{8}$ Jordan, T.L., Langford, W.M., and Hill, J.S. "Airborne Subscale Transport Aircraft Research Testbed: Aircraft Model Development", AIAA 2005-6432, AIAA Guidance, Navigation, and Control Conference and Exhibit, San Francisco, CA, August 2005.

${ }^{9}$ Jordan, T.L., Foster, J.V., Bailey, R.M., and Belcastro, C.M., "AirSTAR: A UAV Platform for Flight Dynamics and Control System Testing," AIAA-2006-3307, 25th AIAA Aerodynamic Measurement Technology and Ground Testing Conference, San Francisco, CA, June 2006.

${ }^{10}$ Murch, A.M., "A Flight Control System Architecture for the NASA AirSTAR Flight Test Facility," AIAA 2008-6990, AIAA Guidance, Navigation, and Control Conference and Exhibit, Honolulu, HI, August 2008.

${ }^{11}$ Cunningham, K., Foster, J.V., Morelli, E.A., and Murch, A.M., "Practical Application of a Subscale Transport Aircraft for Flight Research in Control Upset and Failure Conditions," AIAA-2008-6200, AIAA Atmospheric Flight Mechanics Conference, Honolulu, HI, August 2008.

${ }^{12}$ https://software.nasa.gov/software/LAR-16100-1

${ }^{13}$ http://www.sunflyte.com/SIDBook_SIDPAC.htm

${ }^{14}$ Nguyen, L.T., Ogburn, M.E., Gilbert, W.P., Kibler, K.S., Brown, P.W., and Deal, P.L., "Simulator Study of Stall / PostStall Characteristics of a Fighter Airplane With Relaxed Longitudinal Static Stability", NASA TP 1538, 1979.

${ }^{15}$ Press, W.H., S.A. Teukolsky, W.T. Vettering, and B.R. Flannery Numerical Recipes in FORTRAN: The Art of Scientific Computing, $2^{\text {nd }}$ Ed., Cambridge University Press, New York, NY, 1992.

${ }^{16}$ Morelli, E.A. "High Accuracy Evaluation of the Finite Fourier Transform using Sampled Data," NASA TM 110340, June 1997.

${ }^{17}$ Rabiner, L.R., Schafer, R.W., and Rader, C.M. "The Chirp z-Transform Algorithm and Its Application," The Bell System Technical Journal, Vol. 48, Issue 5, May-June 1969, pp. 1249-1292.

${ }^{18}$ Bendat, J.S. and Piersol, A.G. Engineering Applications of Correlation and Spectral Analysis, $2^{\text {nd }}$ Ed., John Wiley \& Sons, Inc., New York, NY, 1993.

${ }^{19}$ Otnes, R.K. and Enochson, L., Applied Time Series Analysis, Volume 1, Basic Techniques, John Wiley \& Sons, Inc., New York, NY, 1978.

${ }^{20}$ Morelli, E.A. "Flight Test Maneuvers for Efficient Aerodynamic Modeling," Journal of Aircraft, Vol. 49, No. 6, November-December 2012, pp. 1857-1867.

${ }^{21}$ Brandon, J.M. and Morelli, E.A. "Real-Time Global Nonlinear Aerodynamic Modeling from Flight Data," Journal of Aircraft, Vol. 53, No. 5, September-October 2016, pp. 1261-1297.

${ }^{22}$ Morelli, E.A. and Smith, M.S. "Real-Time Dynamic Modeling - Data Information Requirements and Flight Test Results," Journal of Aircraft, Vol. 46, No. 6, November-December 2009, pp. 1894-1905.

${ }^{23}$ Lanczos, C., Applied Analysis, Dover Publications, Inc., New York, NY, 1988.

${ }^{24}$ Morelli, E.A. "Estimating Noise Characteristics from Flight Test Data using Optimal Fourier Smoothing," Journal of Aircraft, Vol. 32, No. 4, July-August 1995, pp. 689-695.

${ }^{25}$ Grauer, J.A., "Random Noise Generation Using Fourier Series," Journal of Aircraft, to be published, available online February 2018.

${ }^{26}$ Gingras, D.R., Barnhart, B., Ranaudo, R., Martos, B., Ratvasky, T.P., and Morelli, E.A. "Development and Implementation of a Model-Driven Envelope Protection System for In-Flight Ice Contamination," AIAA-2010-8141, AIAA Guidance, Navigation, and Control Conference, Toronto, Canada, August 2010; also published as NASA TM-2011-216960, October 2011.

${ }^{27}$ Holzel, M.S. and Morelli, E.A. "Real-Time Frequency Response Estimation from Flight Data," Journal of Guidance, Control, and Dynamics, Vol. 35, No. 5, September-October 2012, pp. 1406-1417.

${ }^{28}$ Grauer, J.A. and Morelli, E.A. "Real-Time Frequency Response Estimation Using Multi-Sine Inputs and Recursive Fourier Transform,” AIAA-2012-4409, AIAA Atmospheric Flight Mechanics Conference, Minneapolis, MN, August 2012. 
${ }^{29}$ Grauer, J.A., Heeg, J., and Morelli, E.A. "Real-Time Frequency Response Estimation of Joined-Wing SensorCraft Aeroelastic Wind Tunnel Data," AIAA-2012-4641, AIAA Atmospheric Flight Mechanics Conference, Minneapolis, MN, August 2012.

${ }^{30}$ Grauer, J.A. and Morelli, E.A. "Method for Real-Time Frequency Response and Uncertainty Estimation," Journal of Guidance, Control, and Dynamics, Vol. 37, No. 1, January-February 2014, pp. 336-343.

${ }^{31}$ Tischler, M.B., Ivler, C.M., and Berger, T. "Comment on 'Method for Real-Time Frequency Response and Uncertainty Estimation," Journal of Guidance, Control, and Dynamics, Vol. 38, No. 3, March 2015, pp. 547-549.

${ }^{32}$ Grauer, J.A. and Morelli, E.A. "Reply by the Authors to M. Tischler, C. Ivler, and T. Berger," Journal of Guidance, Control, and Dynamics, Vol. 38, No. 3, March 2015, pp. 549-550.

${ }^{33}$ Grauer, J.A. "Aircraft Fault Detection Using Real-Time Frequency Response Estimation," AIAA 2016-0372, AIAA Guidance, Navigation, and Control Conference, San Diego, CA, January 2016.

${ }^{34}$ Graham, D. and McRuer, D., Analysis of Nonlinear Control Systems, John Wiley \& Sons, New York, NY, 1961.

${ }^{35}$ Morelli, E.A. "Multiple Input Design for Real-Time Parameter Estimation in the Frequency Domain," Paper REG-360, $13^{\text {th }}$ IFAC Symposium on System Identification, Rotterdam, The Netherlands, August 2003.

${ }^{36}$ Morelli, E.A. "Flight-Test Experiment Design for Characterizing Stability and Control of Hypersonic Vehicles," Journal of Guidance, Control, and Dynamics, Vol. 32, No. 3, May-June 2009, pp. 949-959.

${ }^{37}$ Milliken, W. "Progress in Dynamic Stability and Control Research," Journal of the Aeronautical Sciences, Vol. 14, No. 9, September 1947, pp. 493-519.

${ }^{38}$ Morelli, E.A., "Practical Aspects of the Equation-Error Method for Aircraft Parameter Estimation," AIAA-2006-6144, AIAA Atmospheric Flight Mechanics Conference, Keystone, CO, August 2006.

${ }^{39}$ Shinbrot, M. "On the Analysis of Linear and Nonlinear Dynamical Systems from Transient-Response Data," NACA TN 3288, December 1954.

${ }^{40}$ Pearson, A.E. “Aerodynamic Parameter Estimation Via Fourier Modulating Function Techniques,” NASA CR 4654, April 1995.

${ }^{41}$ Morelli, E.A. "Transfer Function Identification using Orthogonal Fourier Transform Modeling Functions," AIAA 20134749, AIAA Atmospheric Flight Mechanics Conference, Boston, MA, August 2013.

${ }^{42}$ Murphy, P.C. and Klein, V. "Validation of Methodology for Estimating Aircraft Unsteady Aerodynamic Parameters from Dynamic Wind Tunnel Tests," AIAA-2003-5397, AIAA Atmospheric Flight Mechanics Conference and Exhibit, Austin, TX, August 2003.

${ }^{43}$ Greenwell, D.I. “A Review of Unsteady Aerodynamic Modelling for Flight Dynamics of Manoeuvrable Aircraft,” AIAA2004-5276, AIAA Atmospheric Flight Mechanics Conference and Exhibit, Providence, RI, August 2004.

${ }^{44}$ Scharnhorst, K. “Angles in Complex Vector Spaces,” Acta Applicandae Mathematicae, Vol. 69, 2001, pp. 95-103.

${ }^{45}$ Morelli, E.A. "Efficient Global Aerodynamic Modeling from Flight Data," AIAA-2012-1050, 50"th AIAA Aerospace Sciences Meeting, Nashville, TN, January 2012.

${ }^{46}$ Morelli, E.A. and Cooper, J., "Frequency-Domain Method for Automated Simulation Updates based on Flight Data," Journal of Aircraft, Vol. 52, No. 6, November-December 2015, pp. 1995-2008.

${ }^{47}$ Grauer, J.A. "Real-Time Data-Compatibility Analysis Using Output-Error Parameter Estimation," Journal of Aircraft, Vol. 52, No. 3, May-June 2015, pp. 940-947.

${ }^{48}$ Denery, D. “An Identification Algorithm That Is Insensitive to Initial Parameter Estimates," AIAA Journal, Vol. 9, No. 3, March 1971, pp. 371-377.

${ }^{49}$ Taylor, L. and Iliff, K.W. "Systems Identification Using a Modified Newton-Raphson Method - A FORTRAN Program," NASA TN D-6734, May 1972. 
Table 1. Aircraft Geometry and Mass Properties

\begin{tabular}{ccc}
\hline & $\mathrm{F}-16$ & $\mathrm{~T}-2$ \\
\hline length $\bar{c}, \mathrm{ft}$ & 11.32 & 0.908 \\
wing span $b, \mathrm{ft}$ & 30 & 7.083 \\
wing area $S, \mathrm{ft}^{2}$ & 300 & 7.046 \\
$x_{\text {ref }}$ & $0.35 \bar{c}$ & $42.628 \mathrm{in}$ \\
$y_{\text {ref }}$ & 0 & $0.000 \mathrm{in}$ \\
$z_{\text {ref }}$ & 0 & $0.000 \mathrm{in}$ \\
$x_{c g}$ & $0.25 \bar{c}$ & $42.728 \mathrm{in}$ \\
$y_{c g}$ & 0 & $0.000 \mathrm{in}$ \\
$z_{c g}$ & 0 & $0.519 \mathrm{in}$ \\
$m$, slugs & 637.16 & 1.502 \\
$I_{x}$, slugs- $\mathrm{ft}^{2}$ & 9,496 & 1.077 \\
$I_{y}$, slugs- $\mathrm{ft}^{2}$ & 55,814 & 4.163 \\
$I_{z}$, slugs- $\mathrm{ft}^{2}$ & 63,100 & 5.016 \\
$I_{x z},{\text { slugs- } \mathrm{ft}^{2}}^{2}$ & 982 & 0.416 \\
\hline \hline
\end{tabular}

Table 2. Modulating Function Transfer Function Parameter Estimation Results

\begin{tabular}{ccc}
$\begin{array}{c}\text { Parameter } \\
\theta\end{array}$ & True Value & $\begin{array}{c}\text { Estimate } \\
\hat{\theta} \pm \hat{\sigma}\end{array}$ \\
\hline$c_{0}$ & -1.0000 & $-0.9911 \pm 0.0317$ \\
$c_{1}$ & -0.5000 & $-0.4892 \pm 0.0132$ \\
\hline$d_{1}$ & 0.1592 & $0.1566 \pm 0.0037$ \\
$d_{2}$ & 0.0253 & $0.0248 \pm 0.0005$ \\
\hline \hline
\end{tabular}

